\title{
ORTHOGONAL POLYNOMIALS IN CONNECTION WITH QUANTUM GROUPS
}

\author{
TOM H. KOORNWINDER \\ Centre for Mathematics and Computer Science \\ P.O. Box 4079 \\ 1009 AB Amsterdam \\ The Netherlands
}

\begin{abstract}
This is a survey of interpretations of $q$-hypergeometric orthogonal polynomials on quantum groups. The first half of the paper gives general background on Hopf algebras and quantum groups. The emphasis in the rest of the paper is on the $\mathrm{SU}(2)$ quantum group. An interpretation of little q-Jacobi polynomials as matrix elements of its irreducible representations is presented. In the last two sections new results by the author on interpretations of Askey-Wilson polynomials are discussed.
\end{abstract}

\section{Introduction}

Quantum groups, recently introduced by Drinfeld [11], Woronowicz [49] and Jimbo [16], are fascinating objects, where many different structures meet and with applications in numerous areas. In the present paper we want to emphasize one application which was overlooked by the founding fathers of the theory: the interpretation of $q$-hypergeometric orthogonal polynomials, quite analogous to the interpretation of ordinary hypergeometric functions and polynomials on special Lie groups, cf. Vilenkin [47]. So the tremendous amount of new results on $q$-special functions during the last 15 years, cf. Askey and Wilson [6], Gasper and Rahman [14], Rahman [37] is finally matched by a satisfactory "group" theoretic setting. Before the introduction of quantum groups we only knew about interpretations of $q$-Hahn and $q$-Krawtchouk polynomials on Chevalley groups (cf. Stanton [40], [41]) and of $q$-ultraspherical polynomials with $q=0$ on homogeneous trees and $S L_{2}$ over the $p$-adics (cf. Cartier [9]).

Until now, the best studied quantum group is $S U_{q}(2)$, the quantum analogue of the group $S U(2)$. Vaksman and Soibelman [45] were the first to observe that the matrix elements of the irreducible unitary representations of this quantum group can be expressed in terms of little $q$-Jacobi polynomials. The same observation was independently made by Masuda e.a. [27], [28] and by the author [21]. Various other classes of $q$-hypergeometric orthogonal polynomials have been interpreted in connection with $S U_{q}(2)$, see [32], [33], [17], [18] and [22]. In this last reference an addition formula for little $q$-Legendre polynomials is obtained which would have been hard to discover without the interpretation on the quantum group.

Very recently, significant interpretations of special functions have been found on other quantum groups. On the one hand, there are results on more general "compact" quantum 
groups like $S U_{q}(n)$, cf. Noumi e.a. [34]. On the other hand, work is starting now on quantum analogues of non-compact Lie groups like the group of plane motions (cf. Vaksman and Korogodsky [44]) and $S U(1,1)$ (cf. Masuda e.a. [29]).

During the preparation of this manuscript the author obtained an interpretation of continuous $q$-Legendre polynomials as "spherical" matrix elements of irreducible representations of $S U_{q}(2)$. For ordinary $S U(2)$ the definition of spherical depends on the choice of the subgroup, for instance $S(U(1) \times U(1))$ or $S O(2)$, but, still, all one-parameter subgroups are conjugate and thus yield the same spherical functions. However, in the quantum group case the "subgroups" are no longer conjugate and give rise to different types of "spherical functions", for instance little $q$-Legendre and continuous $q$-Legendre polynomials. Next, by a further generalization of the notion of spherical element and with some inspiration from Noumi's and Mimachi's recent preprint [32], a similar interpretation could be obtained for a two-parameter family of Askey-Wilson polynomials. As a spin-off there followed the observation that one can pass from Askey-Wilson polynomials to little or big $q$-Jacobi polynomials by taking suitable limits.

The connection which has thus been made between quantum groups and the "master family" of Askey-Wilson polynomials looks very promising for future research, for instance, for giving a quantum group theoretic proof of the Rahman-Verma [38] addition formula for continuous $q$-ultraspherical polynomials and for interpreting on quantum groups Macdonald's ([24], [25]) $q$-orthogonal polynomials associated with root systems.

The contents of this paper are as follows. Section 2 is a tutorial presenting the basics of Hopf algebras. In section 3 we introduce compact matrix quantum groups, in particular $S U_{q}(2)$. Section 4 deals with the general representation theory, due to Woronowicz, of such quantum groups. In $\S 5$ this is applied to $S U_{q}(2)$, and little $q$-Jacobi polynomials are obtained as matrix elements of the irreducible corepresentations of the corresponding Hopf algebra. Section 6 briefly reviews some further interpretations of special functions on quantum groups. Finally, sections 7 and 8 deal with the new interpretations by the author of Askey-Wilson polynomials on $S U_{q}(2)$, for which full proofs will be published elsewhere. For the case of continuous $q$-Legendre polynomials an idea of the proof is given in $\S 7$ in fairly complete detail. In $\S 8$ the main results for the larger two-parameter family of Askey-Wilson polynomials are just stated.

While reading these expository notes, it may be helpful to consult the tutorials by Rahman [37] and Stanton [41] in these Proceedings.

NOTATION. $\mathbf{Z}_{+}$denotes the set of nonnegative integers.

\section{Hopf Algebras}

Although the term quantum group sounds quite attractive, the name is in fact somewhat misleading: first, because a quantum group is generally not a group, and, second, because the relationship with quantum mechanics is, in my opinion, not as clear and unambiguous as one might wish. If one still wants to get an impression of what is meant by a quantum group, one way would be to study a number of generally accepted examples of quantum groups. Another way, following Drinfeld [11], would be to define a quantum group as the spectrum of a (not necessarily commutative) Hopf algebra. So let us turn to Hopf algebras. 


\subsection{ALGEBRA OF FUNCTIONS ON A GROUP}

The definition of Hopf algebras may overwhelm the uninitiated reader at first confrontation. Therefore, we will start with a detailed discussion of the guiding example: an algebra of functions on a group.

Let $G$ be a group. Thus there is a multiplication $(x, y) \mapsto x y: G \times G \rightarrow G$, a unit element $e \in G$ and a mapping of taking the inverse $x \mapsto x^{-1}: G \rightarrow G$, which together satisfy the well-known group axioms. Let $\mathcal{A}:=\operatorname{Fun}(G)$ be some complex associative algebra with unit consisting of complex-valued functions on the group $G$, where the multiplication $(a, b) \mapsto a b: \mathcal{A} \times \mathcal{A} \rightarrow \mathcal{A}$ and the unit $I \in \mathcal{A}$ are defined pointwise:

$$
(a b)(x):=a(x) b(x), \quad I(x):=1 \quad \text { for all } x \in G
$$

Note that this is a commutative algebra. To start with, one may think about Fun $(G)$ as the algebra of all complex-valued functions on $G$.

The group operations induce certain operations on the algebra $\mathcal{A}$ :

(i) A comultiplication $\Phi: \operatorname{Fun}(G) \rightarrow \operatorname{Fun}(G \times G)$ defined by

$$
(\Phi(a))(x, y):=a(x y), \quad x, y \in G \text {. }
$$

(ii) A counit $e: \operatorname{Fun}(G) \rightarrow \mathrm{C}$ defined by

$$
e(a):=a(e)
$$

(iii) An antipode $\kappa: \operatorname{Fun}(G) \rightarrow \operatorname{Fun}(G)$ defined by

$$
(\kappa(a))(x):=a\left(x^{-1}\right), \quad x \in G .
$$

Note that the mappings $\Phi, e$ and $\kappa$ are unital algebra homomorphisms.

We will rewrite the comultiplication by using tensor products. There is a linear embedding of $\operatorname{Fun}(G) \otimes \operatorname{Fun}(G)$ in $\operatorname{Fun}(G \times G)$ such that

$$
(a \otimes b)(x, y):=a(x) b(y), \quad x, y \in G,
$$

for all $a, b \in \operatorname{Fun}(G)$. Hence also

$$
\left(\sum_{i} a_{i} \otimes b_{i}\right)(x, y)=\sum_{i} a_{i}(x) b_{i}(y) \quad \text { (finite sum). }
$$

(To start with, the tensor products under consideration are algebraic, so they involve only finite sums.) In particular, if $G$ is an algebraic group over $\mathbf{C}$ and if we take for $\mathcal{A}$ the algebra $\operatorname{Pol}(G)$ of all polynomial functions on $G$, then the above linear embedding becomes a linear isomorphism: $\operatorname{Pol}(G) \otimes \operatorname{Pol}(G) \simeq \operatorname{Pol}(G \times G)$. By way of example let $G$ be the algebraic group

$$
S L(2, \mathrm{C}):=\left\{\left(\begin{array}{ll}
x & y \\
u & v
\end{array}\right) \mid x v-y u=1\right\}
$$


Let $\alpha$ be the polynomial function

$$
\alpha:\left(\begin{array}{ll}
x & y \\
u & v
\end{array}\right) \longmapsto x: G \longrightarrow \mathrm{C}
$$

and let similarly $\beta, \gamma, \delta$ be the polynomial functions which map $\left(\begin{array}{ll}x & y \\ u & v\end{array}\right)$ to $y, u, v$, respectively. Then $\operatorname{Pol}(G)$ consists of all functions on $G$ which can be written as polynomials in $\alpha, \beta, \gamma, \delta$.

As another example let $G$ be a compact group and let $\mathcal{A}:=C(G)$, the algebra of all continuous functions on $G$. Then $C(G \times G)$ can be identified with the topological tensor product of $C(G)$ with $C(G)$, i.e., embed the algebraic tensor product of $C(G)$ with $C(G)$ in $C(G \times G)$ as before and then take the completion with respect to the supremum norm on $G \times G$.

Thus, in many cases, we can view the comultiplication $\Phi$ as a unital algebra homomorphism $\Phi: \mathcal{A} \rightarrow \mathcal{A} \otimes \mathcal{A}$. Here $\mathcal{A} \otimes \mathcal{A}$ is an algebra with multiplication such that $(a \otimes b)(c \otimes d)=a c \otimes b d$, and with unit $I \otimes I$.

We can also extend the multiplication in $\mathcal{A}$, which is initially given as the bilinear mapping $(a, b) \mapsto a b: \mathcal{A} \times \mathcal{A} \rightarrow \mathcal{A}$, to the linear mapping

$$
m: \sum_{i} a_{i} \otimes b_{i} \longmapsto \sum_{i} a_{i} b_{i}: \mathcal{A} \otimes \mathcal{A} \longrightarrow \mathcal{A} .
$$

Note that, if $F \in \mathcal{A} \otimes \mathcal{A}$, then

$$
(m(F))(x)=F(x, x) .
$$

We will next rewrite the group axioms for $G$ in terms of axioms for $\Phi, e$ and $\kappa$ :

(i) The associativity $(x y) z=x(y z)$ yields $a((x y) z)=a(x(y z))$ for functions $a$ on $G$. Thus we have the coassociativity axiom

$$
(\Phi \otimes \mathrm{id}) \circ \Phi=(\mathrm{id} \otimes \Phi) \circ \Phi .
$$

(ii) $e x=x=x e$ for $x \in G$. Hence $a(e x)=a(x)=a(x e)$. This yields the counit axiom

$$
(e \otimes \mathrm{id}) \circ \Phi=\mathrm{id}=(\mathrm{id} \otimes e) \circ \Phi .
$$

(iii) If $G$ is a commutative group then $x y=y x$ for all $x, y \in G$. Hence $a(x y)=a(y x)$. Define the linear mapping $\sigma: \mathcal{A} \otimes \mathcal{A} \rightarrow \mathcal{A} \otimes \mathcal{A}$ such that $\sigma(a \otimes b)=b \otimes a$ (the flip automorphism). Then we have, for commutative $G$, the cocommutativity property

$$
\sigma \circ \Phi=\Phi
$$

(iv) $x^{-1} x=e=x x^{-1}$ for $x \in G$. Hence $a\left(x^{-1} x\right)=a(e)=a\left(x x^{-1}\right)$. We will rewrite $a\left(x^{-1} x\right)$ by using $\Phi, \kappa$ and $m$. Observe that

$$
(\Phi(a))(x, y)=a(x y), \text { hence }(((\kappa \otimes \mathrm{id}) \circ \Phi)(a))(x, y)=a\left(x^{-1} y\right) .
$$


Thus

$$
((m \circ(\kappa \otimes \mathrm{id}) \circ \Phi)(a))(x)=(((\kappa \otimes \mathrm{id}) \circ \Phi)(a))(x, x)=a\left(x^{-1} x\right)
$$

We can rewrite $a\left(x x^{-1}\right)$ in a similar way, while $a(e)=e(a) I(x)$. Thus we have the antipode axiom

$$
(m \circ(\kappa \otimes \mathrm{id}) \circ \Phi)(a)=e(a) I=(m \circ(\mathrm{id} \otimes \kappa) \circ \Phi)(a)
$$

The group $G$ can often be recovered from $\operatorname{Fun}(G)$. As a first example consider an algebraic group $G$ and let $\mathcal{A}:=\operatorname{Pol}(G)$, the algebra of polynomial functions on $G$. Then $\chi: \mathcal{A} \rightarrow \mathrm{C}$ is a unital algebra homomorphism if and only if there exists $x \in G$ such that $\chi(a)=a(x)$, and this establishes a one-to-one correspondence $\chi \leftrightarrow x$ between characters $\chi$ of $\mathcal{A}$ and elements $x$ of $G$. If $\chi_{1}(a)=a\left(x_{1}\right)$ and $\chi_{2}(a)=a\left(x_{2}\right)$ then

$$
\left(\left(\chi_{1} \otimes \chi_{2}\right) \circ \Phi\right)(a)=a\left(x_{1} x_{2}\right)
$$

so we can also recover the group multiplication on $G$ from the comultiplication on $\mathcal{A}$.

As a second example let $G$ be a compact group and let $\mathcal{A}:=C(G)$, the algebra of continuous functions on $G$. Then $C(G)$ is a commutative $C^{*}$-algebra with unit, where the *-operation is given by pointwise complex conjugation: $a^{*}(x)=\overline{a(x)}$. The Gelfand theory for commutative $C^{*}$-algebras tells us that $\chi: \mathcal{A} \rightarrow \mathrm{C}$ is a unital $*$-algebra homomorphism if and only if there exists $x \in G$ such that $\chi(a)=a(x)$.

Since $\Phi: \mathcal{A} \rightarrow \mathcal{A} \otimes \mathcal{A}$ is an algebra homomorphism, it is already determined by its action on a set of generators for the algebra $\mathcal{A}$. For instance, if $G:=S L(2, \mathbf{C})$ and $\mathcal{A}:=\operatorname{Pol}(G)$ then the functions $\alpha, \beta, \gamma, \delta$ which send $\left(\begin{array}{ll}x & y \\ u & v\end{array}\right)$ to $x, y, u, v$, respectively, form a set of generators of $\mathcal{A}$. Let us compute, for instance, $\Phi(\alpha)$.

$$
\begin{aligned}
& (\Phi(\alpha))\left(\left(\begin{array}{ll}
x_{1} & y_{1} \\
u_{1} & v_{1}
\end{array}\right),\left(\begin{array}{ll}
x_{2} & y_{2} \\
u_{2} & v_{2}
\end{array}\right)\right)=\alpha\left(\left(\begin{array}{ll}
x_{1} & y_{1} \\
u_{1} & v_{1}
\end{array}\right)\left(\begin{array}{ll}
x_{2} & y_{2} \\
u_{2} & v_{2}
\end{array}\right)\right) \\
& =\alpha\left(\left(\begin{array}{cc}
x_{1} x_{2}+y_{1} u_{2} & * \\
* & *
\end{array}\right)\right)=x_{1} x_{2}+y_{1} u_{2} \\
& =\alpha\left(\left(\begin{array}{ll}
x_{1} & y_{1} \\
u_{1} & v_{1}
\end{array}\right)\right) \alpha\left(\left(\begin{array}{ll}
x_{2} & y_{2} \\
u_{2} & v_{2}
\end{array}\right)\right)+\beta\left(\left(\begin{array}{ll}
x_{1} & y_{1} \\
u_{1} & v_{1}
\end{array}\right)\right) \gamma\left(\left(\begin{array}{ll}
x_{2} & y_{2} \\
u_{2} & v_{2}
\end{array}\right)\right) \\
& =(\alpha \otimes \alpha+\beta \otimes \gamma)\left(\left(\begin{array}{ll}
x_{1} & y_{1} \\
u_{1} & v_{1}
\end{array}\right),\left(\begin{array}{ll}
x_{2} & y_{2} \\
u_{2} & v_{2}
\end{array}\right)\right) .
\end{aligned}
$$

Similarly we compute the action of $\Phi$ on $\beta, \gamma, \delta$. Thus we obtain:

$$
\begin{array}{cc}
\Phi(\alpha)=\alpha \otimes \alpha+\beta \otimes \gamma, & \Phi(\beta)=\alpha \otimes \beta+\beta \otimes \delta, \\
\Phi(\gamma)=\gamma \otimes \alpha+\delta \otimes \gamma, & \Phi(\delta)=\gamma \otimes \beta+\delta \otimes \delta,
\end{array}
$$

and this determines $\Phi(a)$ for all $a \in \mathcal{A}$. 
The above derivation can be done in a more conceptual way if we use the notion of matrix representation, i.e. a homomorphism

$$
\pi: x \longmapsto\left(\begin{array}{ccc}
\pi_{11}(x) & \ldots & \pi_{1 n}(x) \\
\vdots & & \vdots \\
\pi_{n 1}(x) & \ldots & \pi_{n n}(x)
\end{array}\right)
$$

of the group $G$ into the group of invertible complex $n \times n$ matrices. Then the matrix elements $\pi_{i j}$ are functions on $G$. Under further requirements on $\pi$ these functions may be continuous, $C^{\infty}$, analytic, polynomial, etc. Let us consider how $\Phi, e$ and $\kappa$ act on the $\pi_{i j}$ : (i) We have $\pi(x y)=\pi(x) \pi(y)$, hence $\pi_{i j}(x y)=\sum_{k} \pi_{i k}(x) \otimes \pi_{k j}(y)$. Thus

$$
\Phi\left(\pi_{i j}\right)=\sum_{k} \pi_{i k} \otimes \pi_{k j}
$$

(ii) We have $\pi(e)=I$, hence $\pi_{i j}(e)=\delta_{i j}$. Thus

$$
e\left(\pi_{i j}\right)=\delta_{i j}
$$

(iii) We have $\pi\left(x^{-1}\right) \pi(x)=\pi(e)=\pi(x) \pi\left(x^{-1}\right)$, hence

$$
\sum_{k} \pi_{i k}\left(x^{-1}\right) \pi_{k j}(x)=\pi_{i j}(e)=\sum_{k} \pi_{i k}(x) \pi_{k j}\left(x^{-1}\right) .
$$

Thus

$$
\sum_{k} \kappa\left(\pi_{i k}\right) \pi_{k j}=\delta_{i j} I=\sum_{k} \pi_{i k} \kappa\left(\pi_{k j}\right)
$$

In the example of $S L(2, \mathrm{C})$, with $g:=\left(\begin{array}{ll}x & y \\ u & v\end{array}\right)$, we have a two-dimensional matrix representation

$$
g \longmapsto\left(\begin{array}{cc}
\alpha(g) & \beta(g) \\
\gamma(g) & \delta(g)
\end{array}\right)
$$

which explains (2.1) in view of (2.2).

\subsection{DEFINITION OF HOPF ALGEBRA}

The reader may now be sufficiently prepared to digest the general definition of a Hopf algebra. Let $\mathcal{A}$ be a complex linear space. Then $\mathcal{A}$ is a Hopf algebra if the following four properties are satisfied:

(i) $\mathcal{A}$ is an associative algebra with unit $I$, where the multiplication $(x, y) \mapsto x y$ is linearly extended to $m: \mathcal{A} \otimes \mathcal{A} \rightarrow \mathcal{A}$.

(ii) $\mathcal{A}$ is a coassociative coalgebra with comultiplication $\Phi: \mathcal{A} \rightarrow \mathcal{A} \otimes \mathcal{A}$ and counit $e: \mathcal{A} \rightarrow \mathrm{C}$ (linear mappings) satisfying

$$
(\Phi \otimes \mathrm{id}) \circ \Phi=(\mathrm{id} \otimes \Phi) \circ \Phi \text { and }(e \otimes \mathrm{id}) \circ \Phi=\mathrm{id}=(\mathrm{id} \otimes e) \circ \Phi .
$$


(iii) $\mathcal{A}$ is a bialgebra, i.e., $\mathcal{A}$ satisfies both (i) and (ii), and the mappings $\Phi: \mathcal{A} \rightarrow \mathcal{A} \otimes \mathcal{A}$ and $e: \mathcal{A} \rightarrow \mathbf{C}$ are unital algebra homomorphisms.

(iv) There is an antipode $\kappa: \mathcal{A} \rightarrow \mathcal{A}$ (linear mapping) satisfying

$$
(m \circ(\kappa \otimes \mathrm{id}) \circ \Phi)(a)=e(a) I=(m \circ(\mathrm{id} \otimes \kappa) \circ \Phi)(a) \quad \text { for all } a \in \mathcal{A} \text {. }
$$

In general, $\kappa \circ \kappa \neq \mathrm{id}$ ( $\kappa$ is not involutive) and $\kappa(a b) \neq \kappa(a) \kappa(b)$ ( $\kappa$ is not multiplicative). However, it can be shown that

$$
\kappa(a b)=\kappa(b) \kappa(a), \quad \kappa(I)=I, \quad \sigma \circ(\kappa \otimes \kappa) \circ \Phi=\Phi \circ \kappa, \quad e \circ \kappa=e,
$$

so $\kappa$ is an anti-multiplicative unital algebra homomorphism and an anti-comultiplicative counital coalgebra homomorphism.

\subsection{COREPRESENTATIONS OF HOPF ALGEBRAS}

Let $\mathcal{A}$ be a Hopf algebra. A matrix corepresentation of $\mathcal{A}$ is a matrix

$$
u=\left(\begin{array}{ccc}
u_{11} & \ldots & u_{1 n} \\
\vdots & & \vdots \\
u_{n 1} & \ldots & u_{n n}
\end{array}\right) \quad \text { with } u_{i j} \in \mathcal{A}
$$

such that

$$
\Phi\left(u_{i j}\right)=\sum_{k=1}^{n} u_{i k} \otimes u_{k j} \quad \text { and } \quad e\left(u_{i j}\right)=\delta_{i j} .
$$

If $\mathcal{A}=$ Fun $(G)$ for some group $G$ then this definition is equivalent to the definition of matrix representation of $G$. It is possible to give a more abstract definition of corepresentation without use of matrices, for which we refer to the literature.

It follows from the antipode axiom that

$$
\sum_{k=1}^{n} \kappa\left(u_{i k}\right) u_{k j}=\delta_{i j} I=\sum_{k=1}^{n} u_{i k} \kappa\left(u_{k j}\right) .
$$

Two corepresentations $u$ and $v$ of $\mathcal{A}$ are called equivalent if $u$ and $v$ are matrices of the same size $n \times n$ and if there is an invertible complex $n \times n$ matrix $s$ such that

$$
s u=v s \quad \text { (matrix products). }
$$

A corepresentation $u$ of $\mathcal{A}$ is called irreducible if $u$ is not equivalent to a corepresentation $v$ of the form

$$
v=\left(\begin{array}{ll}
* & * \\
0 & *
\end{array}\right)
$$

i.e., if not for some $m, 1 \leq m \leq n-1$, we have $v_{i j}=0$ for all $(i, j)$ such that $m+1 \leq i \leq n$, $1 \leq j \leq m$. 


\subsection{NOTES}

There are two textbooks on Hopf algebras: Sweedler [42] and Abe [1]. A concise introduction to Hopf algebras is given in Hazewinkel $[15, \S 37.1]$. An informal account of some basic facts and examples can be found in Bergman [8]. Manin [26] discusses Hopf algebras in connection with quantum groups. We have introduced notation for Hopf algebra operations as in Woronowicz [49]. In fact, the notation $\Delta$ for comultiplication, $\varepsilon$ for counit and $S$ for antipode is more common, cf. [1], [11]. This last notation will be used in $\S 7$ for dual Hopf algebras, in particular for (quantized) universal enveloping algebras.

\section{Quantum Groups}

In this section we will introduce our main examples of quantum groups: the quantum analogues of the groups $S L(2, \mathrm{C})$ and $S U(2)$. We will also give the definition of Hopf *-algebras and of compact matrix quantum groups.

\subsection{GENERATORS AND RELATIONS}

Many special Hopf algebras are introduced by means of generators and relations. For instance, let $G:=S L(2, \mathrm{C})$ and $\mathcal{A}:=\operatorname{Pol}(G)$, the algebra of polynomial functions on the algebraic group $G$. Then $\mathcal{A}$ as unital algebra is isomorphic to the commutative unital algebra with generators $\alpha, \beta, \gamma, \delta$ and with relation $\alpha \delta-\beta \gamma=I$. We can equivalently describe this algebra as the free commutative unital algebra generated by $\alpha, \beta, \gamma, \delta$ divided out by the (necessarily two-sided) ideal generated by $\alpha \delta-\beta \gamma-I$.

We might also describe $\mathcal{A}$ as the unital algebra with a priori non-commuting generators $\alpha, \beta, \gamma, \delta$ and relations

$$
\begin{aligned}
& \alpha \beta-\beta \alpha=0, \quad \alpha \gamma-\gamma \alpha=0, \quad \beta \delta-\delta \beta=0, \quad \gamma \delta-\delta \gamma=0 \\
& \beta \gamma-\gamma \beta=0, \quad \alpha \delta-\delta \alpha=0, \quad \alpha \delta-\beta \gamma-I=0 .
\end{aligned}
$$

So $\mathcal{A}$ is the free non-commutative unital algebra with generators $\alpha, \beta, \gamma, \delta$ divided out by the two-sided ideal generated by the left hand sides in (3.1).

Note also that the generators are precisely the matrix elements of the corepresentation $\left(\begin{array}{ll}\alpha & \beta \\ \gamma & \delta\end{array}\right)$. This determines the Hopf algebra structure of $\mathcal{A}$ completely.

In general, if a Hopf algebra is presented as algebra by means of generators and relations then it is sufficient to specify $\Phi, e$ and $\kappa$ by their action on the generators, as these operations are multiplicative or anti-multiplicative with respect to the multiplication. If it is already given that the generators are the matrix elements of a corepresentation then the action of $\Phi$ and $e$ on the generators follows by (2.3), while $\kappa$ acting on the generators, if it exists, is uniquely determined by (2.4).

\subsection{THE QUANTUM SL $(2, \mathrm{C})$ GROUP}

This celebrated quantum group is presented by a deformation of the relations (3.1). We will use a deformation parameter $q$, which we will fix at some nonzero complex value. For $q=1$ the Hopf algebra $\operatorname{Pol}(S L(2, \mathbf{C}))$ will be recovered. 
Let $\mathcal{A}_{q}$ be the unital algebra with non-commuting generators $\alpha, \beta, \gamma, \delta$ and with relations

$$
\begin{aligned}
& \alpha \beta=q \beta \alpha, \quad \alpha \gamma=q \gamma \alpha, \quad \beta \delta=q \delta \beta, \quad \gamma \delta=q \delta \gamma, \quad \beta \gamma=\gamma \beta, \\
& \alpha \delta-q \beta \gamma=\delta \alpha-q^{-1} \beta \gamma=I .
\end{aligned}
$$

We define a comultiplication $\Phi$ and a counit $e$ on $\mathcal{A}_{q}$ by the requirement that $\left(\begin{array}{ll}\alpha & \beta \\ \gamma & \delta\end{array}\right)$ is a corepresentation of $\mathcal{A}_{q}$. This yields, by (2.3), again (2.1) for $\Phi$ acting on the generators, and

$$
e(\alpha)=e(\delta)=1, \quad e(\beta)=e(\gamma)=0
$$

Also, if we put

$$
\kappa(\alpha)=\delta, \quad \kappa(\delta)=\alpha, \quad \kappa(\beta)=-q^{-1} \beta, \quad \kappa(\gamma)=-q \gamma,
$$

then (2.4) is satisfied in view of the relations (3.2). The only thing left for the proof that $\mathcal{A}_{q}$ is a Hopf algebra, is to show that the relations (3.2) are preserved by $\Phi$ and $e$ extended as homomorphisms and by $\kappa$ extended as anti-homomorphism. See for instance Woronowicz [50] for a proof that this is indeed the case.

We say that the Hopf algebra $\mathcal{A}_{q}$ is associated with the quantum group $S L_{q}(2, \mathrm{C})$. Observe that $\mathcal{A}_{q}$ is a deformation of the commutative Hopf algebra $\mathcal{A}_{1}=\operatorname{Pol}(S L(2, \mathrm{C}))$ into a non-commutative Hopf algebra. As such, $\mathcal{A}_{q}$ is a kind of quantization of $\mathcal{A}_{1}$, which motivates the name quantum group. Observe also that $S L(2, \mathrm{C})$ is rigid within the category of complex Lie groups, but has a nontrivial deformation within the wider category of quantum groups.

\subsection{HOPF *-ALGEBRAS}

We will now pass from complex to real by introducing a *-operation. Recall that a *-algebra is a complex associative algebra with anti-linear mapping $a \mapsto a^{*}$ which is involutive and anti-multiplicative, i.e. $\left(a^{*}\right)^{*}=a$ and $(a b)^{*}=b^{*} a^{*}$. If the algebra possesses a unit $I$ (which we will always assume) then it is also required that $I^{*}=I$.

A Hopf *-algebra is a Hopf algebra $\mathcal{A}$ with a mapping $a \mapsto a^{*}: \mathcal{A} \rightarrow \mathcal{A}$ such that $\mathcal{A}$ as an algebra becomes a *-algebra, the mappings $\Phi: \mathcal{A} \rightarrow \mathcal{A} \otimes \mathcal{A}$ and $e: \mathcal{A} \rightarrow \mathbf{C}$ are *homomorphisms, and $\kappa$ satisfies

$$
\kappa \circ * \circ \kappa \circ *=\mathrm{id}
$$

The two mappings $\kappa$ and $*$ will not necessarily commute.

A matrix corepresentation $u$ of $\mathcal{A}$ is called unitary if

$$
u_{i j}^{*}=\kappa\left(u_{j i}\right) \text {, }
$$

which can, in view of (2.4), be equivalently written as

$$
\sum_{k} u_{k i}^{*} u_{k j}=\delta_{i j} I=\sum_{k} u_{i k} u_{j k}^{*} .
$$


If $\mathcal{A}$ is presented by generators and relations, then the *-operation is already characterized by its action on the generators. If the generators are moreover the matrix elements of a unitary corepresentation of $\mathcal{A}$ then (3.4) shows how * acts on them.

There is a one-to-one correspondence between the choice of a real form of a complex algebraic group $G$ and the choice of a Hopf *-operation on $\operatorname{Pol}(G)$. For instance, if $\mathcal{A}=$ $\operatorname{Pol}(S L(2, \mathrm{C}))$ then the group

$$
S U(2):=\left\{\left.\left(\begin{array}{cc}
x & -\bar{u} \\
u & \bar{x}
\end{array}\right)|| x\right|^{2}+|u|^{2}=1\right\}
$$

is a compact real form of $S L(2, \mathrm{C})$. Now define $a \mapsto a^{*}$ on $\mathcal{A}$ by first restricting the polynomial $a$ to $S U(2)$, then taking pointwise complex conjugates and finally extending the resulting function to a holomorphic polynomial on $S L(2, \mathrm{C})$. Thus $\alpha^{*}=\delta$ and $\beta^{*}=-\gamma$. On the other hand, the real form $S U(2)$ can be recovered from our knowledge of the *operation: $\chi: \mathcal{A} \rightarrow \mathrm{C}$ is a unital *-homomorphism if and only if there is $x \in S U(2)$ such that $\chi(a)=a(x)$ for all $a \in \mathcal{A}$.

\subsection{THE QUANTUM SU(2) GROUP}

From now on consider the Hopf algebra $\mathcal{A}_{q}$ with $0<q<1$. Often, the results will remain valid for the classical case $q=1$, sometimes the case $q=1$ has to be interpreted by taking a suitable limit. Our restriction is mainly for convenience-the assumption that $q$ is real and nonzero would also be possible.

Let $a \mapsto a^{*}: \mathcal{A}_{q} \rightarrow \mathcal{A}_{q}$ make $\mathcal{A}_{q}$ into a Hopf *-algebra such that $\left(\begin{array}{cc}\alpha & \beta \\ \gamma & \delta\end{array}\right)$ is a unitary corepresentation. This yields

$$
\alpha^{*}=\delta, \quad \delta^{*}=\alpha, \quad \beta^{*}=-q \gamma, \quad \gamma^{*}=-q^{-1} \beta .
$$

It can be verified that the continuation of $*$ to $\mathcal{A}_{q}$ as an anti-linear anti-multiplicative mapping is well-defined in view of the relations (3.2). Thus $\mathcal{A}_{q}$ becomes a Hopf *-algebra, which we say to be associated with the quantum group $S U_{q}(2)$.

\subsection{COMPACT MATRIX QUANTUM GROUPS}

Let us bring a little analysis into this algebraic story. Given a Hopf *-algebra $\mathcal{A}$, generated by the matrix elements of a unitary corepresentation $u$ of $\mathcal{A}$, we want to make $\mathcal{A}$ into a normed *-algebra, by analogy to $\operatorname{Pol}(S U(2))$ with respect to the sup norm.

For a Hilbert space $\mathcal{H}$ let $\mathcal{L}(\mathcal{H})$ be the algebra of all bounded linear operators on $\mathcal{H}$. It is a $\mathrm{C}^{*}$-algebra, as we have $\left\|T T^{*}\right\|=\|T\|^{2}$. By a $*$-representation $\pi$ of $\mathcal{A}$ on $\mathcal{H}$ we mean a homomorphism $\pi: \mathcal{A} \rightarrow \mathcal{L}(\mathcal{H})$ of unital $*$-algebras. Define

$$
\|a\|:=\sup _{\pi}\|\pi(a)\|, \quad \pi \text { running over all *-representations of } \mathcal{A} \text {. }
$$

Since $u$ is a unitary corepresentation, we have $\left\|\pi\left(u_{i j}\right)\right\| \leq 1$ for all $*$-representations $\pi$ and for all indices $i, j$. Hence, for all $a \in \mathcal{A},\|a\|$ will be finite, so $\|\cdot\|$ defines a seminorm on $\mathcal{A}$ and

$$
\left\|a^{*} a\right\|=\|a\|^{2}, \quad a \in \mathcal{A}
$$


If, moreover, $a=0$ whenever $\|a\|=0$, or if, equivalently, $\mathcal{A}$ has a faithful *-representation, then $\|\cdot\|$ is a norm on $\mathcal{A}$ satisfying (3.6). Let $A$ be the norm completion of $\mathcal{A}$ as a normed linear space. Then the *-algebra operations will also extend to $A$ and $A$ will become a $C^{*}$. algebra. Moreover, with a suitable definition of $\mathrm{C}^{*}$-tensor product $A \otimes A, \Phi$ also extends to a $\mathrm{C}^{*}$-homomorphism of $A$ to $A \otimes A$.

We will say that $\mathcal{A}$ is the Hopf *-algebra and $A$ is the Hopf $C^{*}$-algebra associated with a compact matrix quantum group. Here the term matrix quantum group is used because $\mathcal{A}$ is generated by the matrix elements of a corepresentation and the quantum group is called compact because the *-algebra can be made in to a normed *-algebra.

By way of example, let $G$ be a compact Lie group. Equivalently, $G$ is isomorphic to a closed subgroup of $S U(n)$, so we have a faithful unitary matrix representation of $G$. Let $\mathcal{A}$ be the Hopf *-algebra of polynomials in the matrix elements of this representation. Then the above construction makes $\mathcal{A}$ into a normed *-algebra with norm given by the supremum norm on $G$. The $\mathrm{C}^{*}$-algebra completion $A$ of $\mathcal{A}$ can be identified with the (commutative) $\mathrm{C}^{*}$-algebra $\mathrm{C}(\mathrm{G})$ of continuous functions on $G$. As we observed in $\S 2.1$, the group $G$ can be recovered from $A$ by considering the $*$-homomorphisms of $A$ to $C$. In fact, each commutative Hopf $\mathrm{C}^{*}$-algebra fits into this example, cf. [49, Theorem 1.5].

With the above norm and $\mathrm{C}^{*}$-algebra construction we have made contact with Woronowicz' general theory [49] of compact matrix quantum groups. In $\$ 4$ we will state the main results of this theory. However, for special quantu m groups as $S U_{q}(2)$, it is possible to derive such results without reference to the general theory, by only using the Hopf *-algebra structure, cf. Masuda e.a. [28], Vaksman and Soibelman [45].

\subsection{IRREDUCIBLE *-REPRESENTATIONS OF $\mathcal{A}_{q}$}

For a Hopf *-algebra $\mathcal{A}$ associated with a compact matrix quantum group there are two interesting representation theories: the irreducible unitary corepresentations of $\mathcal{A}$ on finite dimensional Hilbert spaces and the irreducible *-representations of $\mathcal{A}$ on possibly infinitedimensional Hilbert spaces. If $\mathcal{A}=\operatorname{Pol}(G)$ with $G$ a compact Lie group, then the irreducible *-representations of $\mathcal{A}$ are one-dimensional and correspond to point evaluations on the elements of $G$. By analogy, for non-commutative $\mathcal{A}$, the irreducible *-representations of $\mathcal{A}$ may be considered as the elements of the underlying quantum group.

Let us consider the classification of the irreducible *-representations of $\mathcal{A}_{q}$, cf. [45]. There is a family of one-dimensional representations $\chi^{\theta}(0 \leq \theta<2 \pi)$ and a family of infinitedimensional representations $\tau^{\theta}(0 \leq \theta<2 \pi)$. The first family is given by

$$
\chi^{\theta}(\alpha)=e^{i \theta}, \quad \chi^{\theta}\left(\alpha^{*}\right)=e^{-i \theta}, \quad \chi^{\theta}(\gamma)=\chi^{\theta}\left(\gamma^{*}\right)=0
$$

The second family is defined on a Hilbert space $\mathcal{H}$ with orthonormal basis $v_{0}, v_{1}, \ldots$ :

$$
\begin{aligned}
& \tau^{\theta}(\gamma) v_{n}=e^{i \theta} \mu^{n} v_{n}, \quad \tau^{\theta}\left(\gamma^{*}\right) v_{n}=e^{-i \theta} \mu^{n} v_{n}, \\
& \tau^{\theta}(\alpha) v_{n}= \begin{cases}\left(1-\mu^{2 n}\right)^{1 / 2} v_{n-1} & \text { if } n>0, \\
0 & \text { if } \mathrm{n}=0,\end{cases} \\
& \tau^{\theta}\left(\alpha^{*}\right) v_{n}=\left(1-\mu^{2 n+2}\right)^{1 / 2} v_{n+1} .
\end{aligned}
$$


Furthermore, it can be shown that the representation $\tau$ defined by the direct integral

$$
\tau:=\int_{0 \leq \theta<2 \pi}^{\oplus} \tau^{\theta} d \theta
$$

is faithful and that $\|a\|=\|\tau(a)\|$ for all $a \in \mathcal{A}$. Finally, each $*$-representation of $\mathcal{A}_{q}$ is a direct integral of irreducible $*$-representations (so is a type I representation).

\subsection{QUANTUM SUBGROUPS}

Let $K:=S(U(1) \times U(1))$ the subgroup of $S U(2)$ of diagonal matrices $\left(\begin{array}{cc}e^{i \theta} & 0 \\ 0 & e^{-i \theta}\end{array}\right)$. This subgroup is isomorphic to $U(1)$. The algebra $\mathcal{B}:=\operatorname{Pol}(K)$ has generators $\alpha, \delta$ (functions evaluating left upper respectively right lower matrix element) with relations $\alpha \delta=\delta \alpha=I$ and *-operation given by $\alpha^{*}=\delta$. There is a comultiplication $\Psi: \mathcal{B} \rightarrow \mathcal{B} \otimes \mathcal{B}$ given by $\Psi(\alpha):=\alpha \otimes \alpha, \Psi(\delta):=\delta \otimes \delta$. Now one way of expressing that $K$ is a closed subgroup of $S U(2)$ is that there is a surjective *-homomorphism $F$ of $\operatorname{Pol}(S U(2)$ ) onto $\operatorname{Pol}(K)$ (namely the restriction of functions on $S U(2)$ to $K$ ), which is moreover intertwining between the comultiplications on the two algebras.

In an analogous way we can consider $K$ as a quantum subgroup of the quantum group $S U_{q}(2)$. The mapping $F: \mathcal{A}_{q} \rightarrow \mathcal{B}$, for which $F(\alpha):=\alpha, F(\delta):=\delta, F(\beta):=F(\gamma):=0$, defines a surjective $*$-homomorphism and the following diagram is commutative:

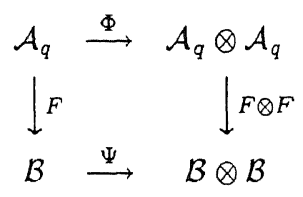

Note that the one-dimensional *-representation $\chi^{\theta}$ applied to $a \in \mathcal{A}_{q}$ is precisely the point evaluation of $F(a)$ at $\operatorname{diag}\left(e^{i \theta}, e^{-i \theta}\right)$.

\subsection{NOTES}

Several motivations have been given for the particular way of defining the $S L(2, \mathbf{C})$ and $S U(2)$ quantum groups, cf. Woronowicz [50, Appendix A1] and Manin [26]. A strong reason for this particular choice is also that the Hopf algebra $\mathcal{A}_{q}$ is contained in the dual Hopf algebra of Jimbo's [16] quantized universal Hopf algebra for root system $A_{1}, \mathrm{cf}$. $\S 7.3$. Thus, in principle, quantum analogues can be constructed of all complex or compact semisimple Lie groups.

See for instance Nijenhuis and Richardson [30] for rigidity of semisimple Lie algebras.

In [27], [28] and [32] the authors write $y, v, u, x$ instead of our $\alpha, \beta, \gamma, \delta$, respectively.

Our use of the term Hopf $C^{*}$-algebras (cf. $\S 3.5$ ) is informal. Different definitions of Hopf $\mathrm{C}^{*}$-algebras have been given in literature, cf. for instance Vallin [46].

Woronowicz [49] called compact matrix quantum groups originally compact matrix pseudogroups. He does not use Hopf algebras, but he starts with a pair $(A, u)$ of a $C^{*}$-algebra $A$ with comultiplication and a corepresentation $u$, such that the matrix elements of $u$ generate a dense *-subalgebra $\mathcal{A}$ of $A$ and an antipode exists on $\mathcal{A}$. Our construction of the Hopf 
$C^{*}$-algebra $A$ from the Hopf *-algebra $\mathcal{A}$ in $§ 3.5$ yields less general $A$ than considered in [49]. For instance, in our approach the counit on $\mathcal{A}$ always extends to $A$, while this is not necessarily the case in [49].

Our definition of Hopf *-algebra in $\S 3.3$ was highly suggested by [49]. The same definition occurs in [44] and a quite similar definition in [26].

In [50] an explicit realization is given of the faithful representation $\tau$ of $\S 3.6$. The operators $\tau^{\theta}\left(\gamma \gamma^{*}\right), \tau^{\theta}(\alpha)$ and $\tau^{\theta}\left(\alpha^{*}\right)$ of $\S 3.6$ form a $q$-analogue of the Schrödinger representation of the Heisenberg algebra and were studied already by Arik and Coon [3] and Feinsilver [13], long before quantum groups were introduced. These authors also considered a $q$-Fock representation. In this connection it is possible to give interpretations of $q$-analogues of Hermite polynomials.

\section{Representation Theory of Compact Matrix Quantum Groups}

Let $\mathcal{A}$ and $A$ be a Hopf *-algebra, respectively Hopf $\mathrm{C}^{*}$-algebra associated with a compact matrix quantum group. In this section we summarize the powerful results of Woronowicz [49] on the representation theory of compact matrix quantum groups, i.e. the corepresentation theory of $\mathcal{A}$ and $A$.

\subsection{HAAR FUNCTIONAL}

The key to harmonic analysis, both on compact groups and on compact matrix quantum groups, is the Haar functional. Recall that, on a compact group $G$, we have a unique measure $d x$, the Haar measure, and corresponding functional $h$ on $C(G)$, the Haar functional

$$
h(a):=\int_{G} a(x) d x, \quad a \in C(G)
$$

with the following properties:

(i) $\int_{G} d x=1$.

(ii) $\int_{G} a(x) d x \geq 0$ if $a \in C(G)$ is nonnegative.

(iii) $\int_{G} a(x y) d x=\int_{G} a(x) d x=\int_{G} a(y x) d x$ if $a \in C(G), y \in G$.

THEOREM 4.1 (Woronowicz [49]). Let $A$ be a Hopf $C^{*}$-algebra. Then there is a unique linear functional $h$ on $A$ such that:

(i) $h(I)=1$.

(ii) $h\left(a^{*} a\right) \geq 0$ for all $a \in A$.

(iii) $\quad(h \otimes \mathrm{id})(\Phi(a))=h(a) I=($ id $\otimes h)(\Phi(a))$ for all $a \in A$.

Moreover, if $a \in A$ and $h\left(a^{*} a\right)=0$ then $a=0$.

Let us give $h$ explicitly on $\mathcal{A}_{q}$. For this we use a certain basis of $\mathcal{A}_{q}$ :

PROPOSITION 4.2 (Woronowicz [50]). The elements $\alpha^{k} \gamma^{m}\left(\gamma^{*}\right)^{n}(k, m, n \geq 0)$ and $\left(\alpha^{*}\right)^{k} \gamma^{m}\left(\gamma^{*}\right)^{n}(k>0, m, n \geq 0)$ form a basis of $\mathcal{A}_{q}$. 
Now we have (cf. Woronowicz [49, Appendix A.1]):

$$
\begin{aligned}
& h\left(\alpha^{k} \gamma^{m}\left(\gamma^{*}\right)^{n}\right)=0=h\left(\left(\alpha^{*}\right)^{k} \gamma^{m}\left(\gamma^{*}\right)^{n}\right) \quad \text { if } k>0 \text { or } m \neq n, \\
& h\left(\left(\gamma \gamma^{*}\right)^{n}\right)=\frac{1-q^{2}}{1-q^{2 n+2}} .
\end{aligned}
$$

Hence, for a polynomial $p$ we have

$$
h\left(p\left(\gamma^{*} \gamma\right)\right)=\left(1-q^{2}\right) \sum_{k=0}^{\infty} p\left(q^{2 k}\right) q^{2 k}=\int_{0}^{1} p(x) d_{q^{2}} x
$$

where we used the notation for $q$-integrals (cf. [37]). However, quite surprisingly, it is also shown in [50, Appendix A.1] that

$$
h\left(p\left(\frac{1}{2}\left(\alpha+\alpha^{*}\right)\right)\right)=\frac{2}{\pi} \int_{-1}^{1} p(x) \sqrt{1-x^{2}} d x
$$

an ordinary integral.

The polynomials in $\gamma \gamma^{*}$ respectively $\left(\alpha+\alpha^{*}\right) / 2$ are the $U(1)$-biinvariant respectively cocentral elements of $\mathcal{A}_{q}$. Here $a$ is called $U(1)$-biinvariant if

$$
\left(\chi^{\theta} \otimes \mathrm{id}\right)(\Phi(a))=a=\left(\mathrm{id} \otimes \chi^{\theta}\right)(\Phi(a)) \quad \text { for all } \theta,
$$

and $a$ is called cocentral if $\sigma \circ \Phi(a)=\Phi(a)$.

\subsection{SCHUR TYPE ORTIIOGONALITY RELATIONS}

Let $G$ be a compact Lie group. Choose for each equivalence class of irreducible unitary representations of $G$ a matrix representation $\left(t_{m n}^{\sigma}\right)_{m, n=1, \ldots, d_{\sigma}}$ as representative. Then the well-known Schur orthogonality relations state that

$$
\int_{G} t_{m k}^{\sigma}(x) \overline{t_{n l}^{\tau}(x)} d x=d_{\sigma}^{-1} \delta_{\sigma \tau} \delta_{m n} \delta_{k l} \text {. }
$$

If, moreover, $G$ is a closed subgroup of some $U(n)$ and if $\mathcal{A}$ is the *-algebra generated by the matrix elements of the natural representation of $G$ then all $t_{m n}^{\sigma}$ are contained in $\mathcal{A}$ and they form a basis of $\mathcal{A}$.

Woronowicz [49] obtained the quantum group analogue of (4.3). In order to formulate this, let $\mathcal{A}$ be a Hopf *-algebra associated with a compact matrix quantum group and choose for each equivalence class of irreducible unitary corepresentations of $\mathcal{A}$ a representative $\left(t_{m n}^{\sigma}\right)_{m, n=1, \ldots, d_{\sigma}}$.

THEOREM 4.3. There exists a unique unital multiplicative linear functional $f$ on $\mathcal{A}$ such that

$$
\begin{aligned}
& h\left(t_{m k}^{\sigma}\left(t_{n l}^{\tau}\right)^{*}\right)=\frac{\delta_{\sigma \tau} \delta_{m n} f\left(t_{l k}^{\sigma}\right)}{f\left(\sum_{l=1}^{d_{\sigma}} t_{l l}^{\sigma}\right)}, \\
& h\left(\left(t_{k m}^{\sigma}\right)^{*} t_{l n}^{\tau}\right)=\frac{\delta_{\sigma \tau} \delta_{m n} \overline{f\left(\left(t_{l k}^{\sigma}\right)^{*}\right)}}{f\left(\sum_{l=1}^{d_{\sigma}} t_{l l}^{\sigma}\right)} .
\end{aligned}
$$

Furthermore, the $t_{m n}^{\sigma}$ form a basis of $\mathcal{A}$. Any finite-dimensional matrix corepresentation of $\mathcal{A}$ is equivalent to a direct sum of irreducible corepresentations of $\mathcal{A}$. 
The occurrence of $f$ in this quantum Schur theorem is a new phenomenon, by which matrix elements belonging to the same representation are no longer orthogonal in a straightforward way. This phenomenon is related to the fact that the Haar functional is not necessarily central, i.e. $h(a b) \neq h(b a)$ in general.

For $\mathcal{A}_{q}$ the unital multiplicative linear functional $f$ was computed in [49, Appendix A.1]. The result is:

$$
f(\alpha)=q^{-1}, \quad f(\delta)=q, \quad f(\beta)=f(\gamma)=0
$$

\section{Little $q$-Jacobi Polynomials Interpreted on $S U_{q}(2)$}

Given an explicit compact matrix quantum group like $S U_{q}(2)$ one may try to realize the following program:

(i) Classify the irreducible unitary corepresentations $t^{\sigma}$.

(ii) Choose suitable bases of the corepresentation spaces, by which one obtains matrix corepresentations $\left(t_{m n}^{\sigma}\right)$.

(iii) Compute the $t_{m n}^{\sigma}$ as polynomials in the generators or other suitable primitives.

(iv) Recognize these polynomials as special functions and rewrite the Schur type orthogonality relations as orthogonality relations for these special functions.

We will discuss this program here for the case $S U_{q}(2)$. Let us first recall the classical case of $S U(2)$, cf. for instance Vilenkin [47, Ch. 3] or Stanton's tutorial [41].

\subsection{IRREDUCIBLE UNITARY REPRESENTATIONS OF SU(2)}

Let $l \in \frac{1}{2} \mathbf{Z}_{+}$. Consider the $(2 l+1)$-dimensional space of homogeneous polynomials of degree $2 l$ in two complex variables, with inner product such that the polynomials

$$
e_{n}^{l}(\xi, \eta):=\left(\begin{array}{c}
2 l \\
l-n
\end{array}\right)^{1 / 2} \xi^{l-n} \eta^{l+n}, \quad n=-l,-l+1, \ldots, l,
$$

form an orthonormal basis. Define a representation $t^{l}$ of $S U(2)$ on this vector space by

$$
\left(t^{l}\left(\begin{array}{ll}
x & y \\
u & v
\end{array}\right) f\right)(\xi, \eta):=f(x \xi+u \eta, y \xi+v \eta)
$$

If $t^{l}$ has matrix elements $t_{m n}^{l}$ with respect to the basis vectors $e_{n}^{l}$ then it follows that

$$
\begin{aligned}
& \left(\begin{array}{c}
2 l \\
l-n
\end{array}\right)^{1 / 2}(x \xi+u \eta)^{l-n}(y \xi+v \eta)^{l+n} \\
& =\sum_{m=-l}^{l} t_{m n}^{l}\left(\left(\begin{array}{ll}
x & y \\
u & v
\end{array}\right)\right)\left(\begin{array}{c}
2 l \\
l-m
\end{array}\right)^{1 / 2} \xi^{l-m} \eta^{l+m} .
\end{aligned}
$$

The basis is such that the matrix $\left(t_{m n}^{l}(g)\right)$ becomes diagonal when $g$ is in the diagonal subgroup $U(1)$ :

$$
t_{m n}^{l}\left(\left(\begin{array}{cc}
e^{i \theta} & 0 \\
0 & e^{-i \theta}
\end{array}\right)\right)=e^{-2 i n \theta} \delta_{m n}
$$


We know that the representations $t^{l}=\left(t_{m n}^{l}\right)$ are unitary and irreducible and that each irreducible unitary representation of $S U(2)$ is equivalent to a $t^{l}$. Furthermore, the $t_{m n}^{l}$ are expressible in terms of Jacobi polynomials, and the Schur orthogonality relations

$$
\int_{G} t_{m n}^{l}(g) \overline{t_{m n}^{\prime \prime}(g)} d g=\frac{\delta_{l, l^{\prime}}}{2 l+1}
$$

are equivalent to orthogonality relations for Jacobi polynomials.

\subsection{IRREDUCIBLE UNITARY COREPRESENTATIONS OF $\mathcal{A}_{q}$}

These can be classified by various methods, cf. Woronowicz [50], Vaksman and Soibelman [45], Masuda e.a. [27], [28], and the author [21]. Here we follow the approach of [21].

Fix $0<q<1$. Let

$$
\left[\begin{array}{l}
n \\
k
\end{array}\right]_{q}:=\frac{(q ; q)_{n}}{(q ; q)_{k}(q ; q)_{n-k}} .
$$

We will use the following lemma, which follows easily by complete induction:

LEMMA 5.1. Let $x y=q y x$. Then

$$
(x+y)^{n}=\sum_{k=0}^{n}\left[\begin{array}{l}
n \\
k
\end{array}\right]_{q} y^{n-k} x^{k}=\sum_{k=0}^{n}\left[\begin{array}{l}
n \\
k
\end{array}\right]_{q^{-1}} x^{k} y^{n-k} .
$$

Let $l, n \in \frac{1}{2} Z_{+}$. By definition of $\Phi$ acting on $\mathcal{A}_{q}$ we have

$$
\begin{aligned}
& \Phi\left(\left[\begin{array}{c}
2 l \\
l-n
\end{array}\right]_{q^{-2}}^{1 / 2} \alpha^{l-n} \gamma^{l+n}\right) \\
& =\left[\begin{array}{c}
2 l \\
l-n
\end{array}\right]_{q^{-2}}^{1 / 2}(\alpha \otimes \alpha+\beta \otimes \gamma)^{l-n}(\gamma \otimes \alpha+\delta \otimes \gamma)^{l+n} .
\end{aligned}
$$

Now expand the right hand side of (5.2) by use of (5.1) and (3.2), such that we get monomials $\alpha^{l-m} \gamma^{l+m}$ in the tensor factors on the right. Then

$$
\begin{aligned}
& {\left[\begin{array}{c}
2 l \\
l-n
\end{array}\right]_{q^{-2}}^{1 / 2}(\alpha \otimes \alpha+\beta \otimes \gamma)^{l-n}(\gamma \otimes \alpha+\delta \otimes \gamma)^{l+n}} \\
& =\sum_{m=-l}^{l} t_{n m}^{l} \otimes\left[\begin{array}{c}
2 l \\
l-m
\end{array}\right]_{q^{-2}}^{1 / 2} \alpha^{l-m} \gamma^{l+m}
\end{aligned}
$$

where the sum runs over $m=-l,-l+1, \ldots, l$ and the $t_{n m}^{l}$ are certain elements of $\mathcal{A}_{q}$. Application of the coassociativity and counit axioms to the left hand side of (5.2) and the right hand side of $(5.3)$ shows that $t^{l}=\left(t_{n m}^{l}\right)$ is a matrix corepresentation of $\mathcal{A}_{q}$. One also sees that $\left(t_{n m}^{l}\right)$ becomes diagonal with respect to the quantum subgroup $U(1)$ :

$$
\chi^{\theta}\left(t_{n m}^{l}\right)=e^{-2 i n \theta} \delta_{n m} .
$$


Formula (5.3) can be considered as a generating function for the matrix elements $t_{n m}^{l}$. From (5.3) another generating function can be derived which sums both over $m$ and $n$.

The coefficients $\left[\begin{array}{c}2 l \\ l-n\end{array}\right]_{q^{-2}}^{1 / 2}$ in $(5.2),(5.3)$ were inserted, because it will turn out that they make the corepresentations unitary. They also make the matrix elements more symmetric, as we will state now. Let $a(\alpha, \beta, \gamma, \delta)$ be some algebraic expression in the generators $\alpha, \beta, \gamma, \delta$ of $\mathcal{A}_{q}$, which yields an element of $\mathcal{A}_{q}$. Let $\tilde{a}(\alpha, \beta, \gamma, \delta)$ be the expression obtained by reversing the order of the factors in all terms of $a(\alpha, \beta, \gamma, \delta)$. Now it follows from the relations (3.2) that the mapping

$$
a(\alpha, \beta, \gamma, \delta) \longmapsto a(\alpha, \gamma, \beta, \delta)
$$

is an algebra isomorphism of $\mathcal{A}_{q}$, while the mapping

$$
a(\alpha, \beta, \gamma, \delta) \longmapsto \tilde{a}(\delta, \beta, \gamma, \alpha)
$$

is an algebra anti-isomorphism of $\mathcal{A}_{q}$. Write $t_{n m}^{l}(\alpha, \beta, \gamma, \delta)$ in order to emphasize that $t_{n m}^{l}$ is some algebraic expression in $\alpha, \beta, \gamma, \delta$. It turns out, just by inspecting (5.3) and the double sum generating function, that the $t_{n m}^{l}$ satisfy the following symmetries:

$$
\begin{aligned}
t_{n m}^{l}(\alpha, \beta, \gamma, \delta) & =t_{m n}^{l}(\alpha, \gamma, \beta, \delta) \\
& =\left(t_{-n,-m}^{l}\right)^{\sim}(\delta, \gamma, \beta, \alpha) \\
& =\left(t_{-m,-n}^{l}\right)^{\sim}(\delta, \beta, \gamma, \alpha) .
\end{aligned}
$$

THEOREM 5.2. The corepresentations $t^{l}$ are unitary and irreducible. Moreover, each irreducible unitary matrix corepresentation of $\mathcal{A}_{q}$ is equivalent to some $t^{l}$.

Here the unitariness follows from (5.4), the irreducibility from reduction to the quantum subgroup $U(1)$ and the nonvanishing of the $t_{n l}^{l}$, the completeness by observing from a somewhat more explicit expression for the $t_{n m}^{l}$ that these elements form a basis of $\mathcal{A}_{q}$.

\subsection{LITTLE $q$-JACOBI POLYNOMIALS}

Recall (cf. [2]) that little $q$-Jacobi polynomials are defined by

$$
p_{n}(x ; a, b ; q):={ }_{2} \phi_{1}\left(\begin{array}{c}
q^{-n}, q^{n+1} a b \\
a q
\end{array} ; q, q x\right)
$$

and that they satisfy orthogonality relations

$$
\begin{aligned}
& \frac{\left(q^{\alpha+1} ; q\right)_{\infty}\left(q^{\beta+1} ; q\right)_{\infty}}{(1-q)(q ; q)_{\infty}\left(q^{\alpha+\beta+2} ; q\right)_{\infty}} \int_{0}^{1} p_{n}(x) p_{m}(x) x^{\alpha} \frac{(q x ; q)_{\infty}}{\left(q^{\beta+1} x ; q\right)_{\infty}} d_{q} x \\
& =\frac{q^{n(\alpha+1)}\left(1-q^{\alpha+\beta+1}\right)\left(q^{\beta+1} ; q\right)_{n}(q ; q)_{n}}{\left(1-q^{2 n+\alpha+\beta+1}\right)\left(q^{\alpha+1} ; q\right)_{n}\left(q^{\alpha+\beta+1} ; q\right)_{n}} \delta_{n m}, \quad \alpha, \beta>-1 .
\end{aligned}
$$

We call the special polynomials $p_{n}(x ; 1,1 ; q)$ little $q$-Legendre polynomials. Now we obtain from (5.3), by straightforward but somewhat tedious computations: 
THEOREM 5.3. We have

$$
t_{n m}^{l}=c_{n m}^{l}\left(\alpha^{*}\right)^{n+m} p_{l-n}\left(\gamma \gamma^{*} ; q^{2(n-m)}, q^{2(n+m)} ; q^{2}\right) \gamma^{n-m},
$$

where $n \geq m \geq-n$ and

$$
c_{n m}^{l}:=\left[\begin{array}{l}
l-m \\
n-m
\end{array}\right]_{q^{2}}^{1 / 2}\left[\begin{array}{c}
l+n \\
n-m
\end{array}\right]_{q^{2}}^{1 / 2} q^{-(n-m)(l-n)} .
$$

This theorem was successively but independently proved by Vaksman and Soibelman [45], Masuda e.a. [27], [28], and the author [21]. Expressions for $t_{n m}^{l}$ in case of the other three possibilities for $n$ and $m$ follow from (5.9) and the symmetries (5.4)-(5.6). In particular, for $l \in \mathbf{Z}_{+}$we have

$$
t_{00}^{l}=p_{l}\left(\gamma \gamma^{*} ; 1,1 ; q^{2}\right)
$$

a little $q$-Legendre polynomial of argument $\gamma \gamma^{*}$.

The Schur type orthogonality (cf. Theorem 4.3 and formula (4.4)) yields

$$
h\left(\left(t_{p r}^{k}\right)^{*} t_{n m}^{l}\right)=\delta_{k l} \delta_{p n} \delta_{r m} \frac{q^{2(l-n)}\left(1-q^{2}\right)}{1-q^{2(2 l+1)}} .
$$

By substitution of (5.9) and (4.1) this is seen to be equivalent to the orthogonality relations (5.8) for little $q$-Jacobi polynomials.

It is also possible to identify the matrix elements as special orthogonal polynomials, when we use (5.12) and have some a priori information about the algebraic structure of the matrix elements. For instance, if we already know that, for $l \in \mathbf{Z}_{+}, t_{00}^{l}$ is a polynomial $p_{l}$ of degree $l$ in $\gamma \gamma^{*}$ then we obtain from (5.12), for $k \neq l$ :

$$
0=h\left(\left(t_{00}^{k}\right)^{*} t_{00}^{l}\right)=\int_{0}^{1} p_{l}(x) p_{k}(x) d_{q^{2}} x
$$

from which we conclude that $p_{l}(x)=$ const. $p_{l}\left(x ; 1,1 ; q^{2}\right)$.

\subsection{NOTES}

Lemma 5.1 is a folk lemma which was often reproved in the literature. In a different formulation it seems to be present already in the works of Netto and MacMahon. It can also be found in Schützenberger [39], Cigler [10] and Feinsilver [12].

In [45], [27] and [28] the second order $q$-difference eigenvalue equation for the little $q$ Jacobi polynomials was obtained from the interpretation on $S U_{q}(2)$ by use of the Casimir element in the dual Hopf algebra to $\mathcal{A}_{q}$.

\section{Summary of Further Interpretations on Quantum Groups}

In this section we briefly indicate some other classes of $q$-hypergeometric orthogonal polynomials and functions which have an interpretation on quantum groups. 


\section{1. $q$-MEIXNER-KRAWTCHOUK POLYNOMIALS}

For fixed $g \in S U(2)$ the representation matrix $\left(t_{m n}^{l}(g)\right)$ dicussed in $\$ 5.1$ is unitary. It is possible to express these matrix elements in terms of Krawtchouk polynomials, such that the orthogonality relations between the rows or columns of the unitary matrix are equivalent to the orthogonality relations for the Krawtchouk polynomials, cf. [31, §12.7] and [20,§2]. In a si milar way (cf. Koornwinder [21]) we can rewrite the orthogonality relations (3.5) for the unitary matrix corepresentation $\left(t_{n m}^{l}\right)$ of $\mathcal{A}_{q}$ as orthogonality relations for the $q$-MeixnerKrawtchouk polynomials

$$
K_{n}\left(q^{-x} ; b, N ; q\right):={ }_{2} \phi_{1}\left(q^{-n}, q^{-x} ; q^{-N} ; q, b q^{n+1}\right), \quad n, x=0,1, \ldots, N
$$

(an ad hoc notation). Before [21] these polynomials had not been recognized in literature as a separate family of orthogonal polynomials. They are $q$-analogues of Krawtchouk polynomials which can be obtained from the $q$-Meixner polynomials by specializing a parameter such that the support of the orthogonality measure becomes finite. Note that the orthogon ality relations (3.5) are identities in $\mathcal{A}_{q}$. These can be made into scalar identities by first rewriting them as operator identities by means of the representations $\tau^{\theta}$ of $\S 3.6$, and next taking matrix elements of these operators with respect to the basis vectors $v_{n}$ of the representation space.

\section{2. $q$-HAIIN and $q$-RACAH POLYNOMIALS}

Clebsch-Gordan coefficients obtained by decomposing the tensor product of two irreducible representations of $S U(2)$ as a direct sum of irreducible representations, can be expressed in terms of Hahn polynomials (cf. for instance [19]). Racah coefficients, which give the transformation between two canonical ways of decomposing a threefold tensor product of irreducible representations of $S U(2)$ as a direct sum of irreducible representations, can be expressed in terms of Racah polynomials (cf. [48]). There are analogous results for $S U_{q}(2)$.

Define the tensor product of two matrix corepresentations $\left(u_{i j}\right)$ and $\left(v_{i j}\right)$ of a Hopf algebra $\mathcal{A}$ as the matrix corepresentation $\left(w_{i k, j l}\right)$, where $w_{i k, j l}:=u_{i j} v_{k l}$. Then, for the tensor product of matrix corepresentations $\left(t_{n m}^{l}\right)$ of $\mathcal{A}_{q}$ we have the direct sum decomposition

$$
t^{l_{1}} \otimes t^{l_{2}}=\bigoplus_{l=l_{1}+l_{2}, l_{1}+l_{2}-1, \ldots,\left|l_{1}-l_{2}\right|} t^{l}
$$

Clebsch-Gordan coefficients for this decomposition were considered by Kirillov and Reshetikhin [17], Vaksman [43] and Koelink and Koornwinder [18]. Here we sketch the approach of [18]. Consider the linear subspace of $\mathcal{A}_{q}$ with basis vectors

$$
\alpha^{l_{1}-n_{1}} \gamma^{l_{1}+n_{1}} \beta^{l_{2}-n_{2}} \delta^{l_{2}+n_{2}}, \quad n_{i}=-l_{i},-l_{i}+1, \ldots, l_{i}, \quad i=1,2 .
$$

There is a natural realization of the corepresentation $t^{l_{1}} \otimes t^{l_{2}}$ on this space. Another basis of this space is given by the matrix elements $t_{n, l_{2}-l_{1}}^{l}$, where $l=l_{1}+l_{2}, l_{1}+l_{2}-1, \ldots,\left|l_{1}-l_{2}\right|$, $n=-l,-l+1, \ldots, l$, and the direct summands in (6.1) have a natural realization on vectors in this second basis. The matrix elements of the transformation matrix from the first to the second basis are called Clebsch-Gordan coefficients. It was shown in [18] that they can be expressed in terms of $q$-Hahn polynomials.

Racah coefficients associated with decompositions of threefold tensor products of corepresentations $t^{l}$ of $\mathcal{A}_{q}$ were studied by Kirillov and Reshetikhin [17]. They expressed them in terms of $q$-Racah polynomials. 


\subsection{ADDITION FORMULA AND WALL POLYNOMIALS}

Let $l \in \mathbf{Z}_{+}, g, h \in S U(2), t^{l}$ as in $§ 5.1$. Then

$$
t_{00}^{l}(g h)=\sum_{k=-l}^{l} t_{0 k}^{l}(g) t_{k 0}^{l}(h) .
$$

By expressing $g$ and $h$ in a suitable way in terms of coordinates on the group, we get the addition formula for Legendre polynomials from this identity, cf. for instance [47, Ch. 3].

Something analogous for $S U_{q}(2)$ was done in Koornwinder [22]. For the corepresentation $t^{l}$ of $\mathcal{A}_{q}$ we have

$$
\Phi\left(t_{00}^{l}\right)=\sum_{k=-l}^{l} t_{0 k}^{l} \otimes t_{k 0}^{l} .
$$

If we substitute (5.9), (5.10) and, in particular (5.11), then we get an identity in $\mathcal{A}_{q} \otimes \mathcal{A}_{q}$ which can be considered as an expansion of the little $q$-Legendre polynomial of degree $l$ and of argument $\Phi\left(\gamma \gamma^{*}\right)$, quite analogous to the addition formula for Legendre polynomials. The passage to a scalar identity is along similar lines as in $\S 6.1$. However, now we have to take matrix elements of operators on $\mathcal{H} \otimes \mathcal{H}$, where $\mathcal{H}$ is the representation space of the representation $\tau^{\theta}$ of $\S 3.6$. These matrix elements are taken on one side with respect to the standard basis, but on the other side with respect to a basis defined by means of Wall polynomials

$$
p_{n}(x ; a, 0 ; q):={ }_{2} \phi_{1}\left(q^{-n}, 0 ; a q ; q, q x\right),
$$

(specialization of little $q$-Jacobi polynomials (5.7)). The resulting addition formula for little $q$-Jacobi polynomials, cf. [22, Theorem 4.1], expands a left hand side

$$
p_{l}\left(q^{x} ; 1,1 ; q\right) p_{y}\left(q^{z} ; q^{x}, 0 ; q\right)
$$

considered as a function of $q^{z}$, in terms of Wall polynomials $p_{y+k}\left(q^{z} ; q^{x}, 0 ; q\right)$. A typical term in the expansion equals

$$
\text { const. } p_{l-k}\left(q^{x+y} ; q^{k}, q^{k} ; q\right) p_{l-k}\left(q^{y} ; q^{k}, q^{k} ; q\right) p_{y+k}\left(q^{z} ; q^{x}, 0 ; q\right) \text {. }
$$

Subsequently, Rahman [36] has given an analytic proof of this addition formula, while van Assche and Koornwinder [7] have shown that the formula tends to the addition formula for Legendre polynomials as $q$ tends to 1 .

In yet unpublished work the author has given a conceptual interpretation of the occurrence of Wall polynomials in the addition formula. It turns out that Wall polynomials occur as Clebsch-Gordan coefficients in the direct integral decomposition for $\tau^{\theta_{1}} \otimes \tau^{\theta_{2}}$.

\subsection{SPHERICAL FUNCTIONS FOR $S U_{q}(n+1) / S U_{q}(n)$}

Recently, Noumi, Yamada and Mimachi [34] announced an interpretation of little $q$-Jacobi polynomials $p_{m}\left(z ; q^{2(n-1)}, q^{2(l-m)} ; q^{2}\right)$ as matrix elements of irreducible corepresentations of the quantum group $S U_{q}(n+1)$ which are biinvariant with respect to the quantum subgroup $S U_{q}(n)$. This result strengthens the expectation that, parallel to the theory of spherical functions on compact symmetric (or other homogeneous) spaces, a similar theory can be developed for quantum groups. 


\subsection{QUANTUM 2-SPHERES AND BIG $q$-JACOBI POLYNOMIALS}

Let $\mathcal{A}$ be a Hopf algebra and $\mathcal{B}$ be an associative algebra. By a coaction of $\mathcal{A}$ on $\mathcal{B}$ we mean a unital algebra homomorphism $\Psi: \mathcal{A} \rightarrow \mathcal{A} \otimes \mathcal{B}$ such that (i) $(\Phi \otimes$ id) $\circ \Psi=($ id $\otimes \Psi) \circ \Psi$ and (ii) $(e \otimes \mathrm{id}) \circ \Psi)=\mathrm{id}$. The guiding example is the case that $G$ is a group acting on a space $X, \mathcal{A}=\operatorname{Fun}(G), \mathcal{B}=\operatorname{Fun}(X)$ and $(\Psi(b))(g, x):=b(g . x)$. If $\mathcal{A}$ is a Hopf *-algebra and $\mathcal{B}$ a $*$-algebra we define a $*$-coaction as a coaction $\Psi$ which is also a $*$-homomorphism. One can think about $\mathcal{A}$ as the dual of a quantum group $G$ and about $\mathcal{B}$ as the dual of a quantum space $X$. Then one has a quantum action of $G$ on $X$. A coaction of $\mathcal{A}$ on $\mathcal{B}$ defines a (usually infinite-dimensional) corepresentation of $\mathcal{A}$ on $\mathcal{B}$. One can try to decompose this into irreducible subspaces.

For the case $S U_{q}(2)$ Podles̀ [35] has defined quantum actions on so called quantum spheres. Next, Noumi and Mimachi [32] have given explicit orthogonal bases for the irreducible subspaces of the algebras corresponding to these quantum spheres. These bases are chosen such that the action of the quantum subgroup $U(1)$ is diagonalized. They obtain big $q$-Jacobi polynomials $P_{n}^{(\alpha, \beta)}(x ; c, d ; q)$ and also, for certain quantum spheres, $q$-Hahn polynomials $Q_{n}\left(x ; q^{\alpha}, q^{\beta}, N ; q\right)$, in both cases with $\alpha=\beta$ and $q$ replaced by $q^{2}$. Next, in [33], Noumi and Mimachi also find a realization for the nonsymmetric case $\alpha \neq \beta$, this time on quantum 3-spheres.

\subsection{QUANTUM GROUP OF PLANE MOTIONS AND $q$-BESSEL FUNCTIONS}

Vaksman and Korogodsky [44] studied the quantum analogue of the group of Euclidean motions of the plane. This work is extremely interesting, since it is the first example of harmonic analysis on a quantum analogue of a noncompact Lie group. Just as the irreducible unitary representations of the group of plane motions have matrix elements expressible in terms of Bessel functions (cf. for instance [47]), so the matrix elements in the quantum case are expressible in terms of $q$-Bessel functions given as ${ }_{1} \phi_{1} q$-hypergeometric series, i.e., different from the more common notion (cf. [14]) of $q$-Bessel functions as $\phi_{1}$ functions.

\subsection{QUANTUM SU(1,1) GROUP}

In [29] Masuda e.a. studied the quantum group $S U_{q}(1,1)$, the quantum analogue of the noncompact semisimple Lie group $S U(1,1)$. The authors obtained series of infinite-dimensional unitary representations of this quantum group. They computed matrix elements of these representations in terms of ${ }_{2} \phi_{1} q$-hypergeometric functions.

\section{8. $q$-EXPONENTIAL FUNCTION}

If $x y=q y x$ then $e_{q}(x+y)=e_{q}(y) e_{q}(x)$, where $e_{q}(x)$ is the $q$-exponential function $\sum_{k=0}^{\infty} x^{k} /(q ; q)_{k}$. This result, due to Schützenberger [39], follows easily from Lemma 5.1. In yet unpublished work the author has shown that the functions $x \mapsto e_{q}(c x)$ occur as one-dimensional representations of a quantum group version of the additive group $\mathbf{R}$ of real numbers. For the construction of this quantum group one needs a slight generalization of the definition of Hopf algebra $\mathcal{A}$. The usual definition of multiplication on $\mathcal{A} \otimes \mathcal{A}$ can be viewed as the mapping $(m \otimes m) \circ(\mathrm{id} \otimes \sigma \otimes \mathrm{id})$ from $\mathcal{A} \otimes \mathcal{A} \otimes \mathcal{A} \otimes \mathcal{A}$ to $\mathcal{A} \otimes \mathcal{A}$. Here $\sigma$ is the flip automorphism. In our generalization we change the definition of this flip. 
Let $\mathcal{A}$ be the free unital algebra generated by one indeterminate $\alpha$. Fix $q \neq 0$. Define

$$
\sigma\left(\alpha^{k} \otimes \alpha^{l}\right):=q^{2 k l} \alpha^{l} \otimes \alpha^{k} .
$$

Then multiplication on $\mathcal{A} \otimes \mathcal{A}$ satisfies

$$
\left(\alpha^{k} \otimes \alpha^{l}\right)\left(\alpha^{m} \otimes \alpha^{n}\right)=q^{2 l m} \alpha^{k+m} \otimes^{l+n} .
$$

We can extend the comultiplication, defined on the generator by

$$
\Phi(\alpha):=\alpha \otimes I+I \otimes \alpha
$$

to an algebra homomorphism $\Phi: \mathcal{A} \rightarrow \mathcal{A} \otimes \mathcal{A}$ by putting

$$
\Phi\left(\alpha^{n}\right):=\sum_{k=0}^{n}\left[\begin{array}{l}
n \\
k
\end{array}\right]_{q^{2}} \alpha^{n-k} \otimes \alpha^{k}
$$

Now $\Phi(a)=a \otimes a$ if $a=e_{q^{2}}(c \alpha)$.

\section{Continuous $q$-Legendre Polynomials}

In the sections 5 and 6 we listed an impressive collection of $q$-hypergeometric orthogonal polynomials admitting an interpretation on the quantum group $S U_{q}(2)$. What we were still badly missing there, are the Askey-Wilson polynomials [6] themselves, i.e., polynomials

$$
\begin{aligned}
p_{n}(\cos \theta ; a, b, c, d \mid q):= & a^{-n}(a b ; q)_{n}(a c ; q)_{n}(a d ; q)_{n} \times \\
& \times{ }_{4} \phi_{3}\left(\begin{array}{c}
q^{-n}, q^{n-1} a b c d, a e^{i \theta}, a e^{-i \theta} \\
a b, a c, a d
\end{array} ; q, q\right),
\end{aligned}
$$

which are, for $q, a, b, c, d \in(-1,1)$, orthogonal polynomials on $(-1,1)$ with respect to a continuous weight function. There is one case where Askey-Wilson polynomials already occurred in connection with $S U_{q}(2)$ : we have the character formula

$$
\operatorname{tr}\left(t^{l}\right):=\sum_{n=-l}^{l} t_{n n}^{l}=U_{2 l}\left(\frac{\alpha+\alpha^{*}}{2}\right)
$$

cf. [49, Appendix A1]. Note that the right hand side is independent of $q$. Here the $U_{n}$ are the Chebysher polynomials of the second kind

$$
U_{n}(\cos \theta):=\frac{\sin (n+1) \theta}{\sin \theta}
$$

These are usually considered as special Jacobi polynomials, but, as pointed out in [6, p.17], they can also be written as special Askey-Wilson polynomials:

$$
U_{n}(\cos \theta)=\frac{p_{n}\left(\cos \theta ; q,-q, q^{1 / 2},-q^{1 / 2} \mid q\right)}{\left(q^{n+2} ; q\right)_{n}}
$$


Note that the left hand side is independent of $q$, although the right hand side would suggest the contrary.

It will turn out that certain Askey-Wilson polynomials can be interpreted as spherical matrix elements of corepresentations $t^{l}$, where the notion spherical has to be specified. I obtained this interpretation first for the continuous $q$-Legendre polynomials, for which we use here the ad hoc notation

$$
\begin{aligned}
P_{n}(\cos \theta \mid q) & :=\frac{p_{n}\left(\cos \theta ; q^{1 / 2},-q^{1 / 2}, q^{1 / 2},-q^{1 / 2} \mid q\right)}{p_{n}\left(\left(q^{1 / 2}+q^{-1 / 2}\right) / 2 ; q^{1 / 2},-q^{1 / 2}, q^{1 / 2},-q^{1 / 2} \mid q\right)} \\
& ={ }_{4} \phi_{3}\left(\begin{array}{c}
q^{-n}, q^{n+1}, q^{1 / 2} e^{i \theta}, q^{1 / 2} e^{-i \theta} \\
q,-q,-q
\end{array} ; q, q\right) .
\end{aligned}
$$

By $[6,(4.20)$ and $(4.2)]$ these polynomials are equal, up to a constant factor, to special continuous $q$-ultraspherical polynomials $C_{n}\left(\cos \theta ; q \mid q^{2}\right)$. They are also Macdonald's ([24], [25]) orthogonal polynomials $P_{\lambda}$ with parameters $q, t$ and associated with root system $A_{1}$ if $t=q^{\frac{1}{2}}$.

I obtained the key for the interpretation on $S U_{q}(2)$ from the formula giving the explicit expansion of $P_{n}(\cos \theta \mid q)$ as a finite Fourier series:

$$
P_{n}(\cos \mid q)=q^{n / 2} \sum_{k=0}^{n} \frac{\left(q ; q^{2}\right)_{k}\left(q ; q^{2}\right)_{n-k}}{\left(q^{2} ; q^{2}\right)_{k}\left(q^{2} ; q^{2}\right)_{n-k}} e^{i(n-2 k) \theta}
$$

cf. $[4,(3.1)]$. The $q=1$ analogue and limit case of this formula is the following finite Fourier series for Legendre polynomials:

$$
P_{n}(\cos \theta)=\sum_{k=0}^{n} \frac{(1 / 2)_{k}(1 / 2)_{n-k}}{k !(n-k) !} e^{i(n-2 k) \theta} .
$$

It turned out that the group theoretic interpretation of (7.3) could be imitated in order to obtain a quantum group theoretic interpretation of (7.2).

\subsection{THE FOURIER SERIES FOR LEGENDRE POLYNOMIALS INTERPRETED}

Put $a_{\theta}:=\left(\begin{array}{cc}e^{i \theta} & 0 \\ 0 & e^{-i \theta}\end{array}\right)$. Recall that, in $\S 5.1$, we considered the representation $\left(t_{m n}^{l}\right)$ of $S U(2)$ with respect to an orthonormal basis $e_{n}^{l}(n=-l,-l+1, \ldots, l)$ of eigenvectors for the $t^{l}\left(a_{\theta}\right)$ :

$$
t^{l}\left(a_{\theta}\right) e_{n}^{l}=e^{-2 i n \theta} e_{n}^{l}
$$

In particular, for $l \in \mathbf{Z}_{+}$:

$$
t^{l}\left(a_{\theta}\right) e_{0}^{l}=e_{0}^{l}
$$

and $e_{0}^{l}$ is, up to a constant factor, the unique $U(1)$-fixed vector in the representation space of $t^{l}$. Now consider the subgroup $K:=S O(2)$ of $S U(2)$. As $U(1)$ and $S O(2)$ are conjugate 
subgroups of $S U(2)$, there must be a $K$-fixed unit vector $e_{K}^{l}$ in the representation space of $t^{l}\left(l \in \mathbf{Z}_{+}\right)$. Expand this vector in terms of the original basis:

$$
e_{K}^{l}=\sum_{n=-l}^{l} c_{n}^{l} e_{n}^{l}
$$

where the $c_{n}^{l}$ are yet unknown.

Put $g:=\left(\begin{array}{cc}x & -\bar{u} \\ u & \bar{x}\end{array}\right)$. The function $g \mapsto P_{l}(x \bar{x})=t_{00}^{l}(g)$ is $U(1)$-biinvariant. By conjugacy the function

$$
g \mapsto P_{l}\left(\frac{1}{2}\left(x^{2}+\bar{x}^{2}+u^{2}+\bar{u}^{2}\right)\right)=\left(t^{l}(g) e_{K}^{l}, e_{K}^{l}\right)=\sum_{n, m=-l}^{l} c_{n}^{l} \overline{c_{m}^{l}} t_{m n}^{l}(g)
$$

is $S O(2)$-biinvariant. For $g:=a_{\theta}$ this yields:

$$
P_{l}(\cos 2 \theta)=\sum_{n=-l}^{l}\left|c_{n}^{l}\right|^{2} e^{-2 i n \theta} \text {. }
$$

So the expansion coefficients in (7.3) follow from the $c_{n}^{l}$ as defined by (7.4). We will find explicit expressions for the $c_{n}^{l}$ by passing to the corresponding Lie algebra representation.

\subsection{AN INFINITESIMAL APPROACH}

The Lie algebra $\mathrm{g}:=\operatorname{sl}(2, \mathrm{C})$ of the Lie group $S L(2, \mathrm{C})$ consists of all complex $2 \times 2$ matrices of trace 0 . It is the complexification of the Lie algebra of $S U(2)$. A basis for $\mathrm{g}$ is given by

$$
H:=\left(\begin{array}{cc}
1 & 0 \\
0 & -1
\end{array}\right), \quad B:=\left(\begin{array}{ll}
0 & 1 \\
0 & 0
\end{array}\right), \quad C:=\left(\begin{array}{ll}
0 & 0 \\
1 & 0
\end{array}\right)
$$

with commutator relations

$$
[H, B]=2 B, \quad[H, C]=-2 C, \quad[B, C]=H .
$$

The representation $t^{l}$ of $g$ corresponding to the representation $t^{l}$ of $S U(2)$ is defined by

$$
t^{l}(X):=\left.\frac{d}{d t}\right|_{t=0} t^{l}(\exp t X)
$$

for $X$ in the Lie algebra of $S U(2)$ and extended to $\mathrm{g}$ by complexification. The explicit expression for $t^{l}$ acting on the basis of $g$ is:

$$
\begin{aligned}
& t^{l}(H) e_{n}^{l}=-2 n e_{n}^{l}, \\
& t^{l}(B) e_{n}^{l}= \begin{cases}\sqrt{(l-n+1)(l+n)} e_{n-1}^{l} & \text { if } n=-l+1,-l+2, \ldots, l, \\
0 & \text { if } n=-l,\end{cases}
\end{aligned}
$$




$$
t^{l}(C) e_{n}^{l}= \begin{cases}\sqrt{(l-n)(l+n+1)} e_{n+1}^{l} & \text { if } n=-l,-l+1, \ldots, l-1, \\ 0 & \text { if } n=l .\end{cases}
$$

The $S O(2)$-invariance of the vector $e_{K}$ (cf. (7.4)) can be infinitesimally characterized as

$$
t^{l}(B-C) e_{K}^{l}=0
$$

When we substitute (7.4), (7.7) and (7.8) in this formula then we obtain a two-term recurrence relation for the $c_{n}^{l}$. Up to a constant factor this can be solved by

$$
c_{n}^{l}= \begin{cases}\left(\frac{(1 / 2)_{(l-n) / 2}(1 / 2)_{(l+n) / 2}}{((l-n) / 2) !((l+n) / 2) !}\right)^{\frac{1}{2}}, & l-n \text { even } \\ 0, & l-n \text { odd }\end{cases}
$$

where $l \in \mathbf{Z}_{+}$. In view of (7.5) this yields (7.3) up to a constant factor.

\subsection{QUANTIZED UNIVERSAL ENVELOPING ALGEBRA}

There is no quantum subgroup of $S U_{q}(2)$ analogous to the subgroup $S O(2)$ of $S U(2)$. However, there is a quantum analogue of the infinitesimal generator $B-C$ of $S O(2)$. For this we need Jimbo's [16] quantization of the universal enveloping algebra of the Lie algebra $s l(2, \mathrm{C})$.

Let $\mathcal{U}_{q}$ be a Hopf algebra with unit 1 , generated as algebra by elements $A, B, C, D$ with relations

$$
\begin{aligned}
& A D=D A=1, \quad A B=q B A, \quad A C=q^{-1} C A, \\
& B C-C B=\frac{A^{2}-D^{2}}{q-q^{-1}} .
\end{aligned}
$$

We can recover (7.6) from (7.11) by substituting in (7.11)

$$
A:=e^{\frac{1}{2}(q-1) H}, \quad D:=e^{-\frac{1}{2}(q-1) H},
$$

and by letting $q$ tend to 1 . We denote the comultiplication by $\Delta: \mathcal{U}_{q} \rightarrow \mathcal{U}_{q} \otimes \mathcal{U}_{q}$. Its action on the generators is given by

$$
\begin{aligned}
& \Delta(A)=A \otimes A, \quad \Delta(D)=D \otimes D \\
& \Delta(B)=A \otimes B+B \otimes D \\
& \Delta(C)=A \otimes C+C \otimes D
\end{aligned}
$$

The counit, denoted by $\varepsilon: \mathcal{U}_{q} \rightarrow \mathrm{C}$, is given by

$$
\varepsilon(A)=\varepsilon(D)=1, \quad \varepsilon(B)=\varepsilon(C)=0 .
$$

The antipode $S: \mathcal{U}_{q} \rightarrow \mathcal{U}_{q}$ is such that

$$
S(A)=D, \quad S(D)=A, \quad S(B)=-q^{-1} B, \quad S(C)=-q C .
$$


It can be easily shown that $\Delta, \varepsilon$ and $S$ have well-defined extensions to $\mathcal{U}_{q}$ as Hopf algebra operations.

We can think about $\mathcal{U}_{q}$ as a dual Hopf algebra to $\mathcal{A}_{q}$, i.e., $\mathcal{U}_{q}$ is embedded in the linear dual of $\mathcal{A}_{q}$ such that the following rules have to be satisfied for $X, Y \in \mathcal{U}_{q}, a, b \in \mathcal{A}_{q}$ :

$$
\begin{aligned}
& (X Y)(a)=(X \otimes Y)(\Phi(a)), \quad(\Delta(X))(a \otimes b)=X(a b), \\
& \varepsilon(X)=X(I), \quad e(a)=a(1), \quad(S(X))(a)=X(\kappa(a)) .
\end{aligned}
$$

In view of the first two rules, it is sufficient to specify $X(a)$ if $X$ is a generator $A, B, C$ or $D$ and $a$ is a generator $\alpha, \beta, \gamma$ or $\delta$. This we declare to yield 0 except in the following cases:

$$
\begin{aligned}
& A(\alpha)=q^{\frac{1}{2}}, \quad A(\delta)=q^{-\frac{1}{2}}, \quad D(\alpha)=q^{-\frac{1}{2}}, \quad D(\delta)=q^{\frac{1}{2}}, \\
& B(\beta)=1, \quad C(\gamma)=1 .
\end{aligned}
$$

It can be shown (cf. Vaksman and Soibelman [45]) that (7.12) and (7.13) yield a well-defined nondegenerate bilinear pairing $\langle X, a\rangle:=X(a)$ between $\mathcal{U}_{q}$ and $\mathcal{A}_{q}$.

The following observation will be important:

$$
\Delta(X)=A \otimes X+X \otimes D \quad \text { if } X=B, C \text { or } A-D
$$

\subsection{CONTINUOUS $q$-LEGENDRE POLYNOMIALS INTERPRETED}

A corepresentation $t^{l}$ of $\mathcal{A}_{q}$ yields a representation of $\mathcal{U}_{q}$ by the rule

$$
t_{n m}^{l}(X):=X\left(t_{n m}^{l}\right)
$$

Indeed, we find that

$$
t_{n m}^{l}(X Y)=\sum_{k=-l}^{l} t_{n k}^{l}(X) t_{k m}^{l}(Y)
$$

Let the vectors $e_{n}^{l}, n=-l,-l+1, \ldots, l$, form the standard basis of the representation space of $t^{l}$, so

$$
t^{l}(X) e_{m}^{l}=\sum_{n=-l}^{l} t_{n m}^{l}(X) e_{n}^{l}
$$

Then the action of $t^{l}$ for the generators of $\mathcal{U}_{q}$ becomes

$$
\begin{aligned}
& t^{l}(A) e_{n}^{l}=q^{-n} e_{n}^{l}, \quad t^{l}(D) e_{n}^{l}=q^{n} e_{n}^{l}, \\
& t^{l}(B) e_{n}^{l}=\frac{\left(q^{-l+n-1}-q^{l-n+1}\right)^{\frac{1}{2}}\left(q^{-l-n}-q^{l+n}\right)^{\frac{1}{2}}}{q^{-1}-q} e_{n-1}^{l}, \\
& t^{l}(C) e_{n}^{l}=\frac{\left(q^{-l+n}-q^{l-n}\right)^{\frac{1}{2}}\left(q^{-l-n-1}-q^{l+n+1}\right)^{\frac{1}{2}}}{q^{-1}-q} e_{n+1}^{l},
\end{aligned}
$$


where, as in (7.8) and (7.11) we suppose $e_{-l-1}^{l}$ and $e_{l+1}^{l}$ to be zero.

In the case of $S U(2)$ we found the $\mathrm{SO}(2)$-invariant vector (7.4) by solving (7.9), which yielded coefficients $c_{n}^{l}$ given by (7.10). We imitate this in the quantum case and look for a solution vector $v:=\sum_{n=-l}^{l} b_{n}^{l, \lambda} e_{n}^{l}$ of

$$
t^{l}\left(q^{\lambda} B-q^{-\lambda} C\right) v=0
$$

where the exponent $\lambda$ is yet to be specified. In view of (7.15) this yields a two-term recurrence relation for the coefficients $b_{n}^{l, \lambda}$, which has only the zero solution if $l \in \frac{1}{2}+\mathbf{Z}_{+}$ and which gives in the case $l \in \mathbf{Z}_{+}$:

$$
v=\text { const. } \sum_{\substack{n=-l \\ l-n \text { even }}}^{l} q^{-\lambda n} c_{n}^{l} e_{n}^{l}
$$

where, for $l \in \mathbf{Z}_{+}$and $n=-l,-l+2, \ldots, l$,

$$
c_{n}^{l}:=\left(\frac{\left(q^{2} ; q^{4}\right)_{(l-n) / 2}\left(q^{2} ; q^{4}\right)_{(l+n) / 2}}{\left(q^{4} ; q^{4}\right)_{(l-n) / 2}\left(q^{4} ; q^{4}\right)_{(l+n) / 2}}\right)^{\frac{1}{2}} .
$$

Now compare with (7.2). We recognize the expansion coefficients in (7.2) as squares of coefficients $c_{n}^{l}$ in (7.17):

$$
P_{l}\left(\cos \theta \mid q^{2}\right)=q^{n} \sum_{\substack{n=-l \\ l-n \text { even }}}^{l}\left(c_{n}^{l}\right)^{2} e^{i n \theta}
$$

This is the crucial observation opening the road to quantum group interpretations of AskeyWilson polynonomials.

A function $a \in \operatorname{Pol}(S U(2))$ is right invariant under $S O(2)$ iff

$$
\left.\frac{d}{d t}\right|_{t=0} a(x \exp (t(B-C)))=0, \quad x \in S U(2),
$$

and left invariant under $S O(2)$ iff

$$
\left.\frac{d}{d t}\right|_{t=0} a(\exp (t(B-C)) x)=0, \quad x \in S U(2) .
$$

We propose as quantum analogues of the conditions (7.19) and (7.20):

$$
\left(\mathrm{id} \otimes\left(q^{\lambda} B-q^{-\lambda} C\right)\right)(\Phi(a))=0,
$$

respectively

$$
\left(\left(q^{\lambda} B-q^{-\lambda} C\right) \otimes \mathrm{id}\right)(\Phi(a))=0 .
$$

Here $\lambda$ is a constant which has yet to be specified. We might say that (7.21) respectively (7.22) express the right respectively left invariance of $a \in \mathcal{A}_{q}$ under the virtual quantum subgroup $S O(2)$.

If $X \in \mathcal{U}_{q}$ then $\Delta(X)$ will be a finite sum of elements of the form $Y \otimes Z$, where $Y, Z \in \mathcal{U}_{q}$. We express this formally as

$$
\Delta(X)=\sum_{(X)} X_{(1)} \otimes X_{(2)}
$$

Then it can easily be shown that: 
LEMMA 7.1. If $\Delta(X)$ is given by (7.23) and $a, b \in \mathcal{A}_{q}$ then

$$
\begin{aligned}
& (\mathrm{id} \otimes X)(\Phi(a b))=\sum_{(X)}\left(\mathrm{id} \otimes X_{(1)}\right)(\Phi(a))\left(\mathrm{id} \otimes X_{(2)}\right)(\Phi(b)) \\
& (X \otimes \mathrm{id})(\Phi(a b))=\sum_{(X)}\left(X_{(1)} \otimes \mathrm{id}\right)(\Phi(a))\left(X_{(2)} \otimes \mathrm{id}\right)(\Phi(b)) .
\end{aligned}
$$

Now it follows from (7.14) that:

PROPOSITION 7.2. The elements $a$ satisfying (7.21) respectively (7.22) form a unital subalgebra of $\mathcal{A}_{q}$.

It is not difficult to show from (7.16) that the elements $a \in \operatorname{Span}\left\{t_{n m}^{l}\right\}$ which satisfy both (7.21) and (7.22), are just the null element if $l \in \frac{1}{2}+\mathbf{Z}_{+}$, and form for $l \in \mathbf{Z}_{+}$a one-dimensional subspace of elements

$$
a=\text { const. } \sum_{\substack{n, m=-l \\ l-n, l-m \text { even }}}^{l} q^{(n-m) \lambda} c_{n}^{l} c_{m}^{l} t_{n m}^{l}
$$

where $c_{n}^{l}$ is given by (7.17). Let us compute (7.24) explicitly for $l=1$. From Theorem 5.3 we get

$$
t_{1,1}^{1}=\left(\alpha^{*}\right)^{2}, \quad t_{2,2}^{1}=\alpha^{2}, \quad t_{1,-1}^{1}=\gamma^{2}, \quad t_{-1,1}^{1}=q^{2}\left(\gamma^{*}\right)^{2}
$$

Thus the element $a \in \operatorname{Span}\left\{t_{n m}^{1}\right\}$ satisfying both (7.21) and (7.22) equals, up to a constant factor,

$$
a=\alpha^{2}+\left(\alpha^{*}\right)^{2}+q^{2 \lambda} \gamma^{2}+q^{2-2 \lambda}\left(\gamma^{*}\right)^{2}
$$

Now we would like to have this element $a$ self-adjoint, i.e., $a=a^{*}$. This forces us to take $\operatorname{Re} \lambda=\frac{1}{2}$. From the point of view of interpretations of special functions, the choice $\lambda:=\frac{1}{2}$ will be sufficient for our purposes. For the moment we call an element $a \in \mathcal{A}_{q}$ spherical if $a$ satisfies (7.21) and (7.22) with $\lambda=\frac{1}{2}$. Put

$$
\rho:=\frac{1}{2}\left(\alpha^{2}+\left(\alpha^{*}\right)^{2}+q \gamma^{2}+q\left(\gamma^{*}\right)^{2}\right)=\rho^{*} .
$$

Then $\rho$ spans the spherical elements in $\operatorname{Span}\left\{t_{n m}^{1}\right\}$. All $a:=p(\rho)$, with $p$ a polynomial, are also spherical. Actually we can prove:

THEOREM 7.3. $a \in \mathcal{A}_{q}$ is spherical if and only if $a$ is of the form $p(\rho)$, with $p$ a polynomial. 
In particular, we can apply this theorem to the spherical elements given by (7.24). Then

$$
\sum_{\substack{n, m=-l \\ l-n, l-m \text { even }}}^{l} q^{(n-m) / 2} c_{n}^{l} c_{m}^{l} t_{n m}^{l}=\sum_{k} b_{k} \rho^{k}
$$

for certain coefficients $b_{k}$. Now apply the characters $\chi^{\theta}$ to both sides of (7.25). This yields

$$
\sum_{\substack{n=-l \\ l-n \text { even }}}^{l}\left(c_{n}^{l}\right)^{2} e^{-2 i n \theta}=\sum_{k} b_{k}(\cos 2 \theta)^{k} .
$$

In view of (7.18) we have

$$
q^{-n} P_{l}\left(\cos 2 \theta \mid q^{2}\right)=\sum_{k} b_{k}(\cos 2 \theta)^{k} .
$$

Hence

$$
q^{-n} P_{l}\left(\rho \mid q^{2}\right)=\sum_{k} b_{k} \rho^{k}
$$

So we obtain:

THEOREM 7.4. Let $l \in \mathbf{Z}_{+}$. Then, up to a constant factor, the spherical element in Span $\left\{t_{n m}^{l}\right\}$ is given by $P_{l}\left(\rho \mid q^{2}\right)$, a continuous $q$-Legendre polynomial of degree $l$ in $\rho$.

We compare next the known orthogonality relations

$$
\begin{aligned}
\frac{\left(q^{4}, q^{4} ; q^{4}\right)_{\infty}}{2 \pi\left(q^{2}, q^{2} ; q^{4}\right)_{\infty}} \int_{0}^{\pi} P_{k}\left(\cos \theta \mid q^{2}\right) P_{l}\left(\cos \theta \mid q^{2}\right) & \left|\frac{\left(e^{2 i \theta} ; q^{4}\right)_{\infty}}{\left(q^{2} e^{2 i \theta} ; q^{4}\right)_{\infty}}\right|^{2} d \theta \\
& =\frac{1-q^{2}}{1-q^{2(2 l+1)}} \delta_{k l}
\end{aligned}
$$

of the continuous $q$-Legendre polynomials with the Schur type orthogonality relations. From (7.25), (7.26) we obtain

$$
P_{l}\left(\rho \mid q^{2}\right)=q^{l} \sum_{\substack{n, m=-l \\ l-n, l-m \text { even }}}^{l} q^{(n-m) / 2} c_{n}^{l} c_{m}^{l} t_{n m}^{l} .
$$

Now substitute (7.28) twice into (7.27) and apply (5.12). This yields

$$
\begin{aligned}
h\left(P_{k}\left(\rho \mid q^{2}\right) P_{l}\left(\rho \mid q^{2}\right)\right) & =\delta_{k l} \frac{\left(1-q^{2}\right) q^{2 l}}{1-q^{2(2 l+1)}}\left(\sum_{\substack{n=-l \\
l-n \text { even }}}^{l} q^{-n}\left(c_{n}^{l}\right)^{2}\right)^{2} \\
& =\delta_{k l} \frac{\left(1-q^{2}\right) q^{2 l}}{1-q^{2(2 l+1)}}\left(P_{l}\left(\left(q+q^{-1}\right) / 2 \mid q^{2}\right)\right)^{2} \\
& =\frac{1-q^{2}}{1-q^{2(2 l+1)}} .
\end{aligned}
$$

By comparing (7.27) with (7.29) we conclude: 
THEOREM 7.5. Let $p$ be a polynomial. Then

$$
h(p(\rho))=\frac{\left(q^{4}, q^{4} ; q^{4}\right)_{\infty}}{2 \pi\left(q^{2}, q^{2} ; q^{4}\right)_{\infty}} \int_{0}^{\pi} p(\cos \theta)\left|\frac{\left(e^{2 i \theta} ; q^{4}\right)_{\infty}}{\left(q^{2} e^{2 i \theta} ; q^{4}\right)_{\infty}}\right|^{2} d \theta
$$

So, beside polynomials in $\gamma^{*} \gamma$ and $\left(\alpha+\alpha^{*}\right) / 2$ (cf. (4.1), (4.2)), the Haar functional can now also be evaluated when acting on polynomials in $\rho$. Note that our derivation of $(7.30)$ is very indirect, without computation of moments as for (4.1).

\section{Askey-Wilson Polynomials}

The theorems in $\S 7$ are not the end of the story. In $\S 6.5$ we mentioned the interpretation by Noumi and Mimachi [32] of big $q$-Jacobi polynomials

$$
P_{n}^{(\alpha, \beta)}(x ; c, d ; q):={ }_{3} \phi_{2}\left(\begin{array}{c}
q^{-n}, q^{n+\alpha+\beta+1}, q^{\alpha+1} x / c \\
q^{\alpha+1},-q^{\alpha+1} d / c
\end{array} ; q, q\right)
$$

with $\alpha=\beta$ on quantum 2-spheres. In particular, they found an interpretation of big $q$ Legendre polynomials $P_{n}^{(0,0)}(x ; c, d ; q)$ as zonal spherical elements on quantum 2 -spheres. It was tempting to relate their results to the approach of $\S 7$. A first link could be made by proving the following

PROPOSITION 8.1. Let $l \in \mathbf{Z}_{+}$and $a \in \operatorname{Span}\left\{t_{n m}^{l}\right\}$. Then $a$ satisfies both

$$
\left(\text { id } \otimes\left(q^{\frac{1}{2}} B-q^{-\frac{1}{2}} C\right)\right)(\Phi(a))=0 \quad \text { ("quantum right } S O(2) \text {-invariance") }
$$

and

$$
\left(\chi^{\theta} \otimes \mathrm{id}\right)(\Phi(a))=a \quad(\text { left } U(1) \text {-invariance })
$$

if and only if

$$
a=\text { const. } P_{l}^{(0,0)}\left(i q^{-1}\left(\alpha \gamma^{*}-\gamma \alpha^{*}\right) ; 1,1 ; q^{2}\right) .
$$

The left $U(1)$-invariance (8.2) can also be expressed by

$$
((A-D) \otimes \mathrm{id})(\Phi(a))=0 .
$$

Recall that (7.14) is also satisfied for $X:=A-D$. This suggests that we may generalize Proposition 8.1 such that we get interpretations of big $q$-Legendre polynomials $P_{n}^{(0,0)}(x ; c, d ; q)$ with $c \neq d$ by keeping $(8.2)$ and replacing $(8.1)$ by

$$
\left(\mathrm{id} \otimes\left(q^{\frac{1}{2}} B-q^{-\frac{1}{2}} C+\text { const. }(A-D)\right)\right)(\Phi(a))=0 .
$$

This was the starting point for some very recent results by the author (yet unpublished), which we will summarize now. 


\section{1. $(\sigma, \tau)$-SPHERICAL ELEMENTS}

Let $\sigma, \tau \in \mathbb{R}$. We will call an element $a \in \mathcal{A}_{q}(\sigma, \tau)$-spherical if

$$
\begin{aligned}
& \left(\mathrm{id} \otimes\left(i q^{\frac{1}{2}} B-i q^{-\frac{1}{2}} C-\frac{q^{-\sigma}-q^{\sigma}}{q^{-1}-q}(A-D)\right)\right)(\Phi(a))=0, \\
& \left(\left(i q^{\frac{1}{2}} B-i q^{-\frac{1}{2}} C-\frac{q^{-\tau}-q^{\tau}}{q^{-1}-q}(A-D)\right) \otimes \mathrm{id}\right)(\Phi(a))=0 .
\end{aligned}
$$

If $\tau= \pm \infty$ then we replace (8.5) by (8.3), and similarly for $\sigma= \pm \infty$. Put

$$
\begin{aligned}
\rho_{\sigma \tau}:= & \frac{1}{2}\left(\alpha^{2}+\left(\alpha^{*}\right)^{2}+q\left(\gamma^{2}+\left(\gamma^{*}\right)^{2}\right)+i q\left(q^{-\sigma}-q^{\sigma}\right)\left(\alpha^{*} \gamma-\gamma^{*} \alpha\right)+\right. \\
& \left.+i q\left(q^{-\tau}-q^{\tau}\right)\left(\alpha^{*} \gamma^{*}-\gamma \alpha\right)-q\left(q^{-\sigma}-q^{\sigma}\right)\left(q^{-\tau}-q^{\tau}\right) \gamma^{*} \gamma\right)=\rho_{\sigma \tau}^{*} .
\end{aligned}
$$

Put, for $l=0,1, \ldots$ and $n=-l,-l+1, \ldots, l$ :

$$
\begin{aligned}
c_{n}^{l, \sigma}:= & \frac{i^{n} q^{-(l+\sigma) n} q^{n^{2} / 2}}{\left(q^{2} ; q^{2}\right)_{l+n}^{1 / 2}\left(q^{2} ; q^{2}\right)_{l-n}^{1 / 2}} \times \\
& \times{ }_{3} \phi_{2}\left(\begin{array}{c}
q^{-2 l+2 n}, q^{-2 l},-q^{-2 l-2 \sigma} \\
q^{-4 l}, 0
\end{array} ; q^{2}, q^{2}\right)=c_{-n}^{l, \sigma} .
\end{aligned}
$$

THEOREM 8.2. Let $a \in \mathcal{A}$. Then $a$ is $(\sigma, \tau)$-spherical if and only if it is a polynomial in $\rho_{\sigma \tau}$.

THEOREM 8.3. Let $l \in \mathbf{Z}_{+}$. The space of $(\sigma, \tau)$-spherical elements in $\operatorname{Span}\left\{t_{n m}^{l}\right\}$ is one-dimensional and spanned by an element which we can represent in the following two ways:

$$
\begin{aligned}
& \sum_{n, m=-l}^{l} q^{(n-m) / 2} c_{m}^{l, \sigma} \overline{c_{n}^{l, \tau}} t_{n m}^{l} \\
& \quad=\frac{c_{l}^{l, \sigma} \overline{c_{l}^{l, \tau}}}{\left.q^{2 l+2} ; q^{2}\right)_{l}} p_{l}\left(\rho_{\sigma \tau} ;-q^{\sigma+\tau+1},-q^{-\sigma-\tau+1}, q^{\sigma-\tau+1}, q^{-\sigma+\tau+1} \mid q^{2}\right) .
\end{aligned}
$$

Here the $p_{l}$ at the right hand side of (8.6) is an Askey-Wilson polynomial (7.1). So we have given a quantum group interpretation of a two-parameter family of Askey-Wilson polynomials.

What about the limit cases as $\sigma$ and/or $\tau$ tend to $\infty$ ? We should get little or big $q$-Jacobi polynomials, but these latter polynomials have discrete orthogonality measures, while the Askey-Wilson polynomials have absolutely continuous orthogonality measure, at least as the parameters stay within $(-1,1)$. However, some parameters of the Askey-Wilson polynomials in (8.6) tend to $\infty$ as $\sigma$ or $\tau$ tend to $\infty$. Then discrete mass points are added, cf. [6, Theorems 2.4, 2.5]. If we make, at the same time, a scale transformation, such that the continuous spectrum shrinks, then we will arrive in the limit at infinitely many mass 
points and no continuous spectrum left. In fact, it follows immediately from (7.1), with the normalization

$$
\tilde{p}_{n}(x ; a, b, c, d \mid q):=\frac{p_{n}(x ; a, b, c, d \mid q)}{p_{n}\left(\left(a+a^{-1} / 2\right) ; a, b, c, d \mid q\right)}
$$

that we have the two following limits:

$$
\begin{aligned}
& \tilde{p}_{n}\left(\frac{-x}{2 q^{(a+b-1) / 2}} ;-q^{(1+a+b) / 2},-q^{(1-a-b) / 2}, q^{(1+a-b) / 2}, q^{(1-a+b) / 2} \mid q\right) \\
& \stackrel{a \rightarrow \infty}{\longrightarrow} 3 \phi_{2}\left(\begin{array}{c}
q^{-n}, q^{n+1}, q x \\
q,-q^{b+1}
\end{array} ; q, q\right)=\text { big } q \text {-Jacobi polynomial }
\end{aligned}
$$

and

$$
\begin{aligned}
& \tilde{p}_{n}\left(\frac{-x}{2 q^{a-1 / 2}} ;-q^{1 / 2+a},-q^{1 / 2-a}, q^{1 / 2}, q^{1 / 2} \mid q\right) \\
& \stackrel{a \rightarrow \infty}{\longrightarrow}{ }_{3} \phi_{2}\left(\begin{array}{c}
q^{-n}, q^{n+1}, q x \\
q, 0
\end{array}, q, q\right)=\text { little } q \text {-Jacobi polynomial. }
\end{aligned}
$$

R. Askey told me that he has known such limit transitions already for several years, but never published them.

We also get new expressions for the Haar functional. Let $d m(x)=d m_{a, b, c, d ; q}(x)$ be the normalized orthogonality measure for the Askey-Wilson polynomials:

$$
\int_{-1}^{1}\left(p_{m} p_{n}\right)(x ; a, b, c, d \mid q) d m(x)=\delta_{m n} \frac{\left(1-q^{-1} a b c d\right)(q, a b, a c, a d, b c, b d, c d ; q)_{n}}{\left(1-q^{2 n-1} a b c d\right)\left(q^{-1} a b c d ; q\right)_{n}} .
$$

THEOREM 8.4. Let $p$ be a polynomial. Let $h$ be the Haar functional on $\mathcal{A}$. Then

$$
h\left(p\left(\rho_{\sigma \tau}\right)\right)=\int_{0}^{1} p(x) d m_{a, b, c, d ; q^{2}}(x),
$$

where $a=-q^{\sigma+\tau+1}, b=-q^{-\sigma-\tau+1}, c=q^{\sigma-\tau+1}, d=q^{-\sigma+\tau+1}$.

By putting $\sigma=\tau=0$ in the Theorems 8.2-8.4, we get back the results of $\S 7.4$. If we put $\sigma=\tau$ and let $\sigma \rightarrow \infty$ then we approach, by (8.8), the little $q$-Legendre case. If we fix $\sigma$ and let $\tau \rightarrow \infty$ then, by (8.7), we approach the big $q$-Jacobi case. It should be possible to relate this last case to the results in [32].

\subsection{DUAL $q$-KRAWTCHOUK POLYNOMIALS INTERPRETED}

It is possible to give an explicit matrix for the transition in the representation space of $t^{l}$ from the basis of eigenvectors $e_{n}^{l}$ for $t^{l}(A-D)$ to a basis of eigenvectors for $t^{l}\left(H_{\sigma}\right)$, where

$$
H_{\sigma}:=D\left(i q^{1 / 2} B-i q^{-1 / 2} C-\frac{q^{-\sigma}-q^{\sigma}}{q^{-1}-q}(A-D)\right) .
$$


(Observe that (8.4) can be written as (id $\left.\otimes H_{\sigma}\right)(\Phi(a))=0$ and that $H_{\sigma}$ is self-adjoint if we make $\mathcal{U}_{q}$ into a $*$-algebra such that $A^{*}=A, D^{*}=D, B^{*}=C$. Then $t^{l}$ is a $*$-representation of $\mathcal{U}_{q}$.)

We define dual $q$-Krawtchouk polynomials by

$$
R_{n}\left(q^{-x}-q^{x-N-c} ; q^{c}, N \mid q\right):={ }_{3} \phi_{2}\left(q^{-n}, q^{-x},-q^{x-N-c} ; 0, q^{-N} ; q, q\right) .
$$

These are special $q$-Racah polynomials and satisfy the orthogonality relations

$$
\begin{aligned}
& \frac{1}{\left(-q^{c} ; q\right)_{N}} \sum_{x=0}^{N}\left(R_{n} R_{m}\right)\left(q^{-x}-q^{x-N-c} ; q^{c}, N \mid q\right) \times \\
& \times \frac{\left(1+q^{2 x-N-c}\right)\left(-q^{-N-c}, q^{-N} ; q\right)_{x}}{\left(1+q^{-N-c}\right)\left(q,-q^{-c-1} ; q\right)_{x}\left(-q^{x-2 N-c}\right)^{x}}=\delta_{n m} \frac{(q ; q)_{n}}{\left(q^{-N} ; q\right)_{n}}\left(-q^{-N-c}\right)^{n},
\end{aligned}
$$

where $n, m=0, \ldots, N$. See Askey and Wilson [5] and Stanton [40].

THEOREM 8.5. $t^{l}\left(H_{\sigma}\right)$ has simple spectrum consisting of eigenvalues

$$
x_{j}:=\frac{q^{2 j-\sigma}-q^{\sigma-2 j}+q^{\sigma}-q^{-\sigma}}{q^{-1}-q}, \quad j=-l,-l+1, \ldots, l .
$$

An eigenvector corresponding to eigenvalue $x_{j}$ is given by

$$
\begin{aligned}
\sum_{n=0}^{2 l} i^{-n} q^{n \sigma} q^{n(n+1) / 2}\left(q^{2} ; q^{2}\right)_{n}^{-1 / 2} & \left(q^{4 l} ; q^{-2}\right)_{n}^{1 / 2} \times \\
& \times R_{n}\left(q^{-2 l-2 j}-q^{2 j-2 l-2 \sigma} ; q^{2 \sigma}, 2 l \mid q^{2}\right) e_{n-l}^{l} .
\end{aligned}
$$

Noumi and Mimachi told me that, in a follow-up to [32], they have also obtained such an interpretation of $q$-Krawtchouk polynomials.

\section{References}

[1] E. Abe, Hopf Algebras, Cambridge University Press, 1980.

[2] G. E. Andrews an R. Askey, 'Enumeration of partitions: the role of Eulerian series and q-orthogonal polynomials', pp. 3-26 in Higher Combinatorics (M. Aigner, ed.), Reidel, 1977.

[3] M. Arik and D. D. Coon, 'Hilbert spaces of analytic functions and generalized coherent states', J. Math. Phys. 17 (1976), 524-527.

[4] R. Askey and M. E. H. Ismail, 'A generalization of ultraspherical polynomials', pp. 55-78 in Studies in Pure Mathematics (P. Erdös, ed.), Birkhäuser, 1983.

[5] R. Askey and J. Wilson, 'A set of orthogonal polynomials that generalize the Racah coefficients or 6-j symbols', SIAM J. Math. Anal. 10 (1979), 1008-1016.

[6] R. Askey and J. Wilson, Some basic hypergeometric orthogonal polynomials that generalize Jacobi polynomials, Memoirs Amer. Math. Soc. 54 (1985) No. 319. 
[7] W. Van Assche and T. H. Koornwinder, Asymptotic behaviour for Wall polynomials and the addition formula for little $q$-Legendre polynomials, preprint, 1989.

[8] G. M. Bergman, 'Everybody knows what a Hopf algebra is', Contemp. Math. 43 (1985), 25-48.

[9] P. Cartier, Harmonic analysis on trees, Proc. Sympos. Pure Math. 26 (1973), 419-424.

[10] J. Cigler, 'Operatormethoden für q-Identitäten', Monatsh. Math. 88 (1979), 87-105.

[11] V. G. Drinfeld, 'Quantum groups', pp. 798-820 in Proceedings of the International Congress of Mathematicians, Berkeley, 1986, American Mathematical Society, 1987.

[12] Ph. Feinsilver, 'Commutators, anti-commutators and Eulerian calculus', Rocky Mountain J. Math. 12 (1982), 171-183.

[13] Ph. Feinsilver, 'Discrete analogues of the Heisenberg-Weyl algebra', Monatsh. Math. 104 (1987), 89-108.

[14] G. Gasper and M. Rahman, Basic hypergeometric series, Cambridge University Press, 1989.

[15] M. Hazewinkel, Formal groups and applications, Academic Press, 1978.

[16] M. Jimbo, 'A $q$-difference analogue of $U(\mathbf{g})$ and the Yang-Baxter equation', Lett. Math. Phys. 10 (1985), 63-69.

[17] A. N. Kirillov and N. Yu. Reshetikhin, Representations of the algebra $U_{q}(s l(2)), q-$ orthogonal polynomials and invariants of links, LOMI Preprints E-9-88, Leningrad, 1988.

[18] H. T. Koelink and T. H. Koornwinder, 'The Clebsch-Gordan coefficients for the quantum group $S_{\mu} U(2)$ and $q$-Hahn polynomials', Nederl. Akad. Wetensch. Proc. Ser. A, to appear.

[19] T. H. Koornwinder, 'Clebsch-Gordan coefficients for $S U(2)$ and Hahn polynomials', Nieuw Archief Wisk. (3) 29 (1981), 140-155.

[20] T. H. Koornwinder, 'Krawtchouk polynomials, a unification of two different group theoretic interpretations', SIAM J. Math. Anal. 13 (1982), 1011-1023.

[21] T. H. Koornwinder, 'Representations of the twisted $S U(2)$ quantum group and some $q$-hypergeometric orthogonal polynomials', Nederl. Akad. Wetensch. Proc. Ser. A 92 (1989), 97-117.

[22] T. H. Koornwinder, The addition formula for little $q$-Legendre polynomials and the $S U(2)$ quantum group, CWI Rep. AM-R8906, preprint, 1989.

[23] T. H. Koornwinder, 'Continuous $q$-Legendre polynomials are spherical matrix elements of irreducible representations of the quantum $S U(2)$ group', CWI Quarterly, to appear.

[24] I. G. Macdonald, Orthogonal polynomials associated with root systems, preprint, 1988.

[25] I. G. Macdonald, 'Orthogonal polynomials associated with root systems', These Proceedings.

[26] Yu. I. Manin, Quantum groups and non-commutative geometry, Centre de Recherches Mathématiques, Montréal, 1988.

[27] T. Masuda, K. Mimachi, Y. Nakagami, M. Noumi and K. Ueno, 'Representations of quantum groups and a $q$-analogue of orthogonal polynomials', C. R. Acad. Sci. Paris, Sér. I Math. 307 (1988), 559-564. 
[28] T. Masuda, K. Mimachi, Y. Nakagami, M. Noumi and K. Ueno, 'Representations of the quantum group $S U_{q}(2)$ and the little $q$-Jacobi polynomials', J. Functional Anal., to appear.

[29] T. Masuda, K. Mimachi, Y. Nakagami, M. Noumi, Y. Saburi and K. Ueno, Unitary representations of the quantum group $S U_{q}(1,1), I, I I$, preprint, 1989.

[30] A. Nijenhuis and R. W. Richardson, 'Deformations of Lie algebra structures', J. Math. Mech. 17 (1967), 89-105.

[31] A. F. Nikiforov and V. B. Ugarov, Special Functions of Mathematical Physics, Birkhäuser, 1988.

[32] M. Noumi and K. Mimachi, Quantum 2-spheres and big $q$-Jacobi polynomials, preprint, 1989.

[33] M. Noumi and K. Mimachi, Big $q$-Jacobi polynomials, $q$-Hahn polynomials and a family of quantum 3-spheres, preprint, 1989.

[34] M. Noumi, H. Yamada and K. Mimachi, Zonal spherical functions on the quantum homogeneous space $S U_{q}(n+1) / S U_{q}(n)$, Proc. Japan Acad. 65 (1989), 169-171.

[35] P. Podles̀, 'Quantum spheres', Lett. Math. Phys. 14 (1987), 193-202.

[36] M. Rahman, A simple proof of Koornwinder's addition formula for the little $q$-Legendre polynomials, preprint, 1988.

[37] M. Rahman, 'Some extensions of the beta integral and the hypergeometric function', These Proceedings.

[38] M. Rahman and A. Verma, 'Product and addition formula for the continuous $q$-ultraspherical polynomials', SIAM J. Math. Anal. 17 (1986), 1461-1474.

[39] M. P. Schützenberger, 'Une interprétation de certaines solutions de l'équation fonctionnelle: $F(x+y)=F(x) F(y)$ ', C. R. Acad. Sci. Paris 236 (1953), 352-353.

[40] D. Stanton, 'Orthogonal polynomials and Chevalley groups' pp. 87-128 in Special Functions: Group Theoretic Aspects and Applications (R. A. Askey, T. H. Koornwinder and W. Schempp, eds.), Reidel, 1984.

[41] D. Stanton, 'An introduction to group representations and orthogonal polynomials', These Proceedings.

[42] M. E. Sweedler, Hopf Algebras, Benjamin, 1969.

[43] L. L. Vaksman, $q$-Analogues of Clebsch-Gordan coefficients in the algebra of functions on the quantum group $S U(2), 1989$.

[44] L. L. Vaksman and L. I. Korogodsky, 'Algebra of bounded functions on the quantum group of plane motions and $q$-analogues of Bessel functions', Dokl. Akad. Nauk SSSR 304 (1989), 1036-1040 (in Russian).

[45] L. L. Vaksman and Ya. S. Soibelman, 'Algebra of functions on the quantum group $S U(2)$ ', Functional Anal. Appl. 22 (1988), 170-181.

[46] J.-M. Vallin, 'C*-algèbres de Hopf et $\mathrm{C}^{*}$-algèbres de Kac', Proc. London Math. Soc. (3) 50 (1985), 131-174.

[47] N. Ya. Vilenkin, Special Functions and the Theory of Group Representations, Amer. Math. Soc. Transl. of Math. Monographs, Vol. 22, 1968. 
[48] J. A. Wilson, 'Some hypergeometric orthogonal polynomials', SIAM J. Math. Anal. $11(1980), 690-701$.

[49] S. L. Woronowicz, 'Compact matrix pseudogroups', Comm. Math. Phys. 111 (1987), 613-665.

[50] S. L. Woronowicz, 'Twisted $S U(2)$ group. An example of a non-commutative differential calculus', Publ. Res. Inst. Math. Sci. 23 (1987), 117-181. 